\title{
Fundamentals of reservoir surface energy as related to surface properties, wettability, capillary action, and oil recovery from fractured reservoirs by spontaneous imbibition
}

\author{
DE-FC26-03NT15408
Quarterly Report
01/01/2005 - 03/31/2005 \\ Norman R. Morrow, Principal Investigator \\ Herbert Fischer \\ Yu Li \\ Geoffrey Mason \\ Douglas Ruth \\ Siddhartha Seth \\ Peigui Yin \\ Shaochang Wo
}

April 2005

Submitted by:

Chemical \& Petroleum Engineering

University of Wyoming

Dept. 3295, 1000 E. University Ave

Laramie, WY 82071 


\section{Disclaimer}

This report was prepared as an account of work sponsored by an agency of the United States Government. Neither the United States Government nor any agency thereof, nor any of their employees, makes any warranty, express or implied, or assumes any legal liability or responsibility for the accuracy, completeness, or usefulness of any information, apparatus, product, or process disclosed, or represents that its use would not infringe privately owned rights. Reference herein to any specific commercial product, process, or service by trade name, trademark, manufacturer, or otherwise does not necessarily constitute or imply its endorsement, recommendation, or favoring by the United States Government or any agency thereof. The views and opinions of authors expressed herein do not necessarily state or reflect those of the United States Government or any agency thereof. 


\section{ABSTRACT}

The objective of this project is to increase oil recovery from fractured reservoirs through improved fundamental understanding of the process of spontaneous imbibition by which oil is displaced from the rock matrix into the fractures. Spontaneous imbibition is fundamentally dependent on the reservoir surface free energy but this has never been investigated for rocks. In this project, the surface free energy of rocks will be determined by using liquids that can be solidified within the rock pore space at selected saturations. Thin sections of the rock then provide a two-dimensional view of the rock minerals and the occupant phases. Saturations and oil/rock, water/rock, and oil/water surface areas will be determined by advanced petrographic analysis and the surface free energy which drives spontaneous imbibition will be determined as a function of increase in wetting phase saturation. The inherent loss in surface free energy resulting from capillary instabilities at the microscopic (pore level) scale will be distinguished from the decrease in surface free energy that drives spontaneous imbibition.

A mathematical network/numerical model will be developed and tested against experimental results of recovery versus time over broad variation of key factors such as rock properties, fluid phase viscosities, sample size, shape and boundary conditions. Two fundamentally important, but not previously considered, parameters of spontaneous imbibition, the capillary pressure acting to oppose production of oil at the outflow face and the pressure in the nonwetting phase at the no-flow boundary versus time, will also be measured and modeled. Simulation and network models will also be tested against special case solutions provided by analytic models.

In the second stage of the project, application of the fundamental concepts developed in the first stage of the project will be demonstrated. The fundamental ideas, measurements, and analytic/numerical modeling will be applied to mixed-wet rocks. Imbibition measurements will include novel sensitive pressure measurements designed to elucidate the basic mechanisms that determine induction time and drive the very slow rate of spontaneous imbibition commonly observed for mixed-wet rocks. In further demonstration of concepts, three approaches to improved oil recovery from fractured reservoirs will be tested; use of surfactants to promote imbibition in oil wet rocks by wettability alteration: manipulation of injection brine composition: reduction of the capillary back pressure which opposes production of oil at the fracture face. 


\section{TABLE OF CONTENTS}

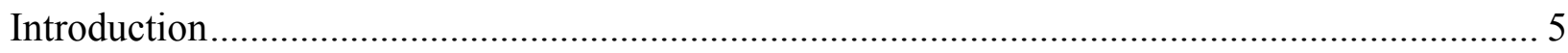

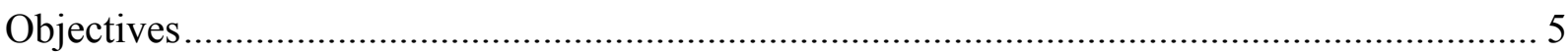

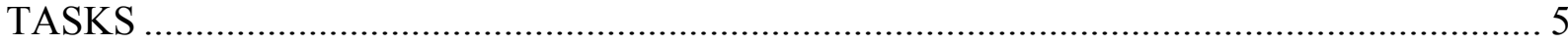

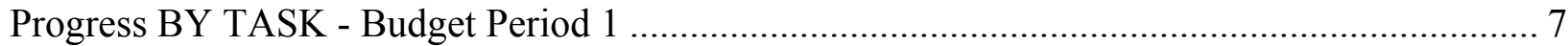

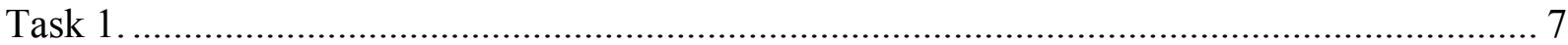

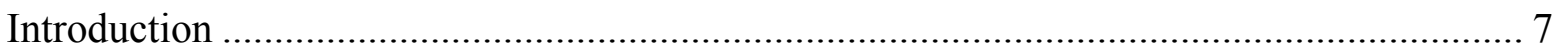

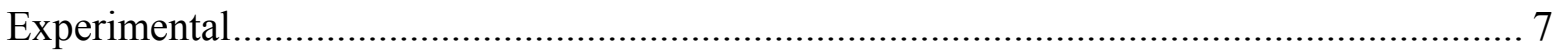

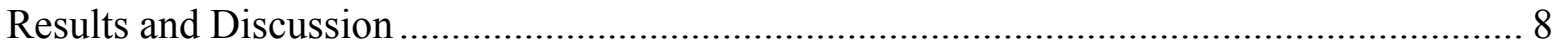

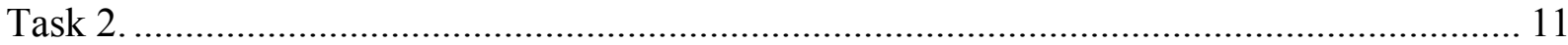

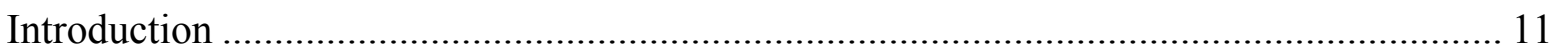

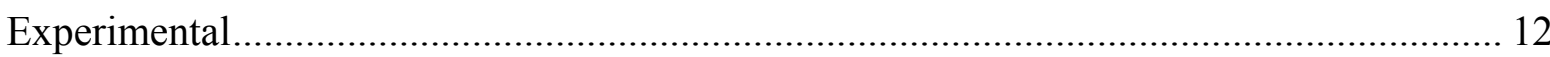

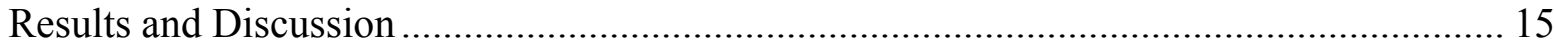

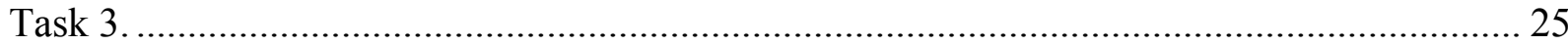

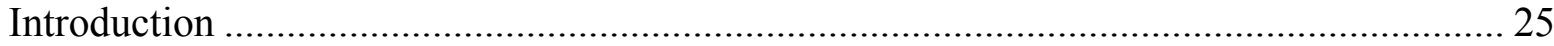

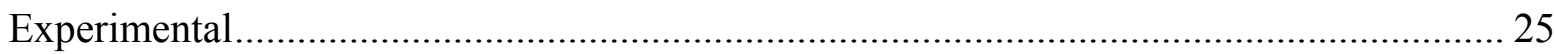

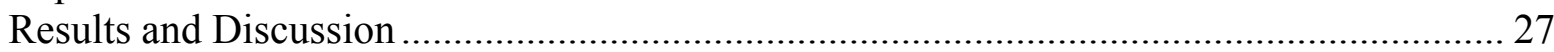

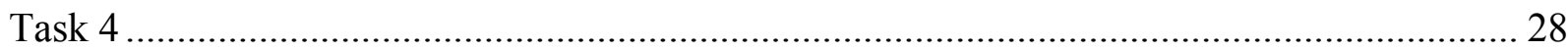

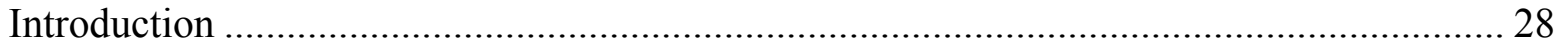

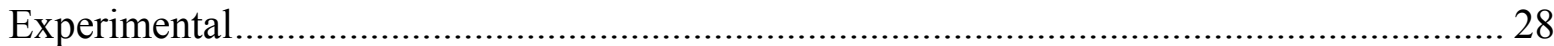

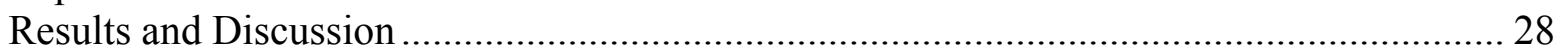

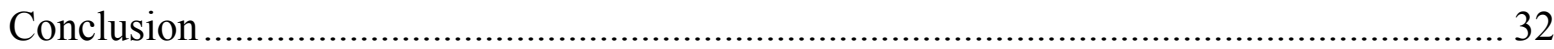

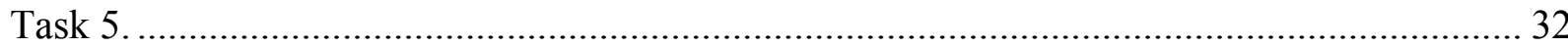

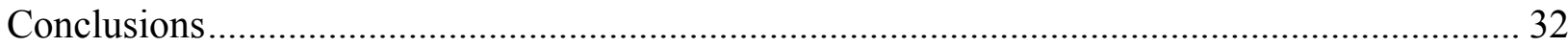

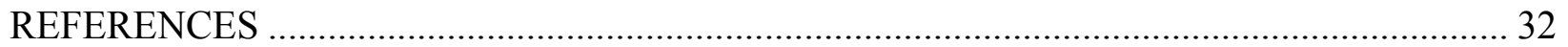




\section{INTRODUCTION}

\section{Objectives}

The long-range objective of this project is to improve oil recovery from fractured reservoirs through improved fundamental understanding of the process of spontaneous imbibition by which oil is displaced from the rock matrix into the fractures. Spontaneous imbibition is fundamentally dependent on the surface energy. An initial objective is to determine the surface energy and relate the dissipation of surface energy to the mechanism of spontaneous imbibition. A parallel objective is to model the mechanism of spontaneous imbibition by a combination of network analysis and numerical modeling. Also fundamentally important, but not previously considered, parameters of spontaneous imbibition, the capillary pressure acting to oppose production of oil at the outflow face and the pressure in the nonwetting phase at the no-flow boundary (in effect within oil in the non-invaded zone of the rock matrix) versus time, will also be measured and compared with values predicted by the mathematical model. The next objective is to measure surface energy and related spontaneous imbibition phenomena for mixed-wettability rocks prepared by adsorption from crude oil. The dissipation of surface free energy must then be related to oil production at mixed-wet conditions. The final objective is to apply the results of the project to improved oil recovery from fractured reservoirs in three ways: reduction of the capillary force that opposes oil production at the fracture face; change in wettability towards increased water wetness; identification of conditions where choice of invading brine composition can give improved recovery.

\section{TASKS}

\section{Budget period 1, July 1, 2003 through June 30, 2005 - Ideas and Concept development: Fundamentals of Spontaneous Imbibition}

Task 1. Work of displacement and surface free energy. Obtain complementary sets of capillary pressure drainage and imbibition data and data on changes in rock/brine, rock/oil, and oil/brine interfacial areas with change in saturation for drainage and imbibition for at least two rock types (sandstone and carbonate). Determine free-energy/work-of-displacement efficiency parameters for drainage and imbibition for at least two rock types so that changes in rock/wetting phase/nonwetting phase surface areas can be closely estimated from capillary pressure measurements.

Task 2. Imbibition in simple laboratory and mathematical network models. Study imbibition in at least three simple tube networks that can be modeled analytically to establish and/or confirm fundamental aspects of the pore scale mechanism of dynamic spontaneous imbibition with special emphasis on determining how spontaneous imbibition is initiated and the key factors in how the saturation profile develops with time. Incorporate rules developed from laboratory measurements on relatively simple networks into the design of a computational network model. Use the network model to obtain an account of the mechanism by which imbibition is initiated, the saturation profile is developed, and the rate of spontaneous imbibition in terms of the dissipation of surface free energy that accompanies change in saturation. 
Task 3. Novel observations on fluid pressures during imbibition and the mechanism of nonwetting phase production at the imbibition face. Make novel observations on the imbibition mechanism including details of the mechanism of oil production at the outflow rock face and the change in the nonwetting phase pressure at the no-flow boundary of the core during the course of spontaneous imbibition for at least 16 distinct combinations of rock/ fluid properties.

Task 4. Network/numerical model and new imbibition data. Develop a numerical simulator specifically designed for spontaneous imbibition. Incorporate the network model to obtain a network/numerical model that includes matching the measured pressure in the nonwetting phase at the no-flow boundary, and the pressure that opposes production of oil at the open rock face. Imbibition data will be obtained for at least 10 rocks with over six-fold variation in permeability, and at least 6 orders of magnitude variation in viscosity ratio, and at least 10 variations in sample size, shape, and boundary conditions.

Task 5. Comparison with similarity solutions. Compare results given by simulation with special case analytic results given by similarity solutions for spontaneous imbibition for at least five distinct cases of rock and fluid properties.

Budget Period 2, July 1, 2005 through June 30, 2008 - Demonstration of concept: Application to mixed wettability rocks and improved oil recovery from fractures reservoirs.

Task 6. Rock preparation and Work of displacement and surface areas

Obtain a range of rock types and identify and obtain crude oils that induce stable mixed wettability. Prepare at least 25 rocks with mixed wettability through crude oil/brine/rock interactions.

Determine work of displacement for drainage and imbibition and measure the variation in rock/brine, rock oil, and oil/brine interfacial areas during the course of drainage and imbibition for at least two examples of mixed wettability.

Task 7. Novel imbibition measurements on mixed-wet rock and network models. Obtain, for at least six mixed-wet rocks, spontaneous imbibition data that includes measurements of the nonwetting phase pressure at the no-flow boundary, observations on the capillary pressure that resists production at the open rock face.

Task 8. Application of network/numerical model to mixed wet rocks. Use network models to relate dissipation of surface energy to rate of spontaneous imbibition and to account for the frequently observed induction time prior to the onset of spontaneous imbibition into mixed wettability rocks.

Task 9. Increased oil recovery by spontaneous imbibition. The mechanism of increased recovery from mixed wet rocks by use of surfactants that promote spontaneous imbibition by favorable wettability alteration will be investigated for at least four distinct examples of crude oil/brine/rock/surfactant combinations.

The mechanism of increased recovery by manipulation of brine composition will be investigated for at least four crude oil/brine/rock combinations. 
Addition of very low concentration surfactants to the imbibing aqueous phase will be explored as a means of increasing the rate of oil recovery by reducing the capillary forces which resist production of oil at the fracture face. At least twelve combinations of rock and fluid properties including both very strongly wetted and mixed wet rocks will be tested.

\section{PROGRESS BY TASK - BUDGET PERIOD 1}

\section{Task 1. Work of displacement and surface free energy.}

\section{Introduction}

All porous media exhibit some residual wetting phase saturation when drained. In the case of a random pack of uniform sized spheres, similar to those used by Morrow (1970), the capillary pressure curve is universal with a well defined irreducible wetting phase saturation. At the irreducible saturation, there is a surface area associated with the wetting phase that provides a measure of the efficiency of conversion of work of displacement to the surface energy created during the drainage process. The work of drainage will always exceed surface free energy because the drainage mechanism is inherently irreversible. In the case of a glass bead pack, the efficiency of displacement was reported to be $79 \%$. For a more complex geometry, such as a sedimentary rock, wetting phase does not usually give a well defined irreducible saturation because water paths in corners, at rough surfaces, and through micropores allow slow drainage. The International Union of Pure Applied Chemistry defined the different types of pores as follows:

$$
\begin{aligned}
& \text { Micropores: } \mathrm{r}<2 \mathrm{~nm} \\
& \text { Mesopores: } \mathrm{r}=2-53 \mathrm{~nm} \\
& \text { Macropores: } \mathrm{r}>53 \mathrm{~nm}
\end{aligned}
$$

\section{Experimental}

The work of displacement was calculated using the area under the capillary pressure curve that can be obtained from mercury injection, centrifuge, porous plate or any other technique.

$$
\text { Work of displacement }=\mathrm{V}_{\mathrm{b}} * \phi \int_{100}^{S w i} P_{c} * d S_{w}
$$

Where, $\mathrm{P}_{\mathrm{c}}$ is the capillary pressure

$\mathrm{S}_{\mathrm{w}}$ is the saturation of the wetting phase

$\mathrm{V}_{\mathrm{b}}$ is the volume of the bulk phase

$\phi$ is the porosity of the sample

Work done under the capillary pressure was used to calculate the area corresponding to it:

$$
\mathrm{A}_{\text {mercury }}=\mathrm{V}_{\mathrm{b}} * \phi \int_{100}^{S w i} P_{c} * d S_{w} /\left(\sigma_{\mathrm{w}-\mathrm{nw}}\right) \quad 1.2
$$
$\mathrm{dyn} / \mathrm{cm})$

$\sigma_{\mathrm{w}-\mathrm{nw}}$ is the interfacial tension between the wetting and the non wetting phase (485 
Alpak et al. (1999) used the area under the capillary pressure curve as an estimate of the surface area of the rock, in theory the work done is not equal to the change in surface energy of the system (in case of rocks) and hence surface area predicted by equating the two would be larger than the actual area created.

\section{Results and Discussion}

\section{PETROPHYSICAL PROPERTIES OF THE ROCK Permeability and porosity}

The air permeability and the porosity of the sandstone sample (PH2) was 740 md and $22 \%$ respectively.

\section{SURFACE AREA AND PORE SIZE DISTRIBUTION ANALYSIS \\ Mercury injection}

Mercury injection has been widely used to estimate capillary pressure and pore size distribution for porous media. Mercury porosimetry is based on the Washburn (1921) equation. This method has the advantage of producing fast results over a large range of pore radii (10 $\mathrm{nm}$ $200 \mu \mathrm{m})$. Fig 1.1 shows the raw capillary pressure curve that was obtained by mercury injection. The kink observed in the data at $\mathrm{S}_{\mathrm{w}} \sim 20 \%$ was due to switching from the low pressure to the high pressure cell during the experiment. At the beginning of the experiment the mercury fills up areas of contact between the core and the core holder. This raw data was corrected for the entry region using the following procedure (shown diagrammatically in Fig 1.2)

1. Two tangents to the curve were made one for the flat portion and other for the entry region (T1 and $\mathrm{T} 2$ )

2. A line was drawn from second data point at $\mathrm{S}_{\mathrm{w}} \sim 95 \%$ (T3)

3. The mid point of T1 andT3 was normalized to $S_{\mathrm{w}}=100 \%$

4. The pink in the data at $S_{w} \sim 20 \%$ was smoothed.

The final smoothed capillary pressure curve that was used for obtaining the work done and the depth of investigation is shown in Fig 1.3. The area obtained corresponding to the work done calculated using Eq 1.2 is shown in Fig 1.4. Fig 1.4 conclusively shows that the amount of surface created by the invading non wetting phase (mercury) was a small fraction of the surface area obtained by the BET surface area analysis. At a residual wetting phase saturation of $\mathrm{S}_{\mathrm{w}} \sim 5$ $\%$ the total area created is $0.11 \mathrm{~m}^{2} / \mathrm{g}$ which was about $\sim 17 \%$ of the BET surface area $(0.626$ $\mathrm{m}^{2} / \mathrm{g}$ ). The reason for this difference is that most of the BET surface area in the case of the PH2 sample is due to microporous region that is not invaded by non wetting phase, even though the pore space is $95 \%$ filled. 


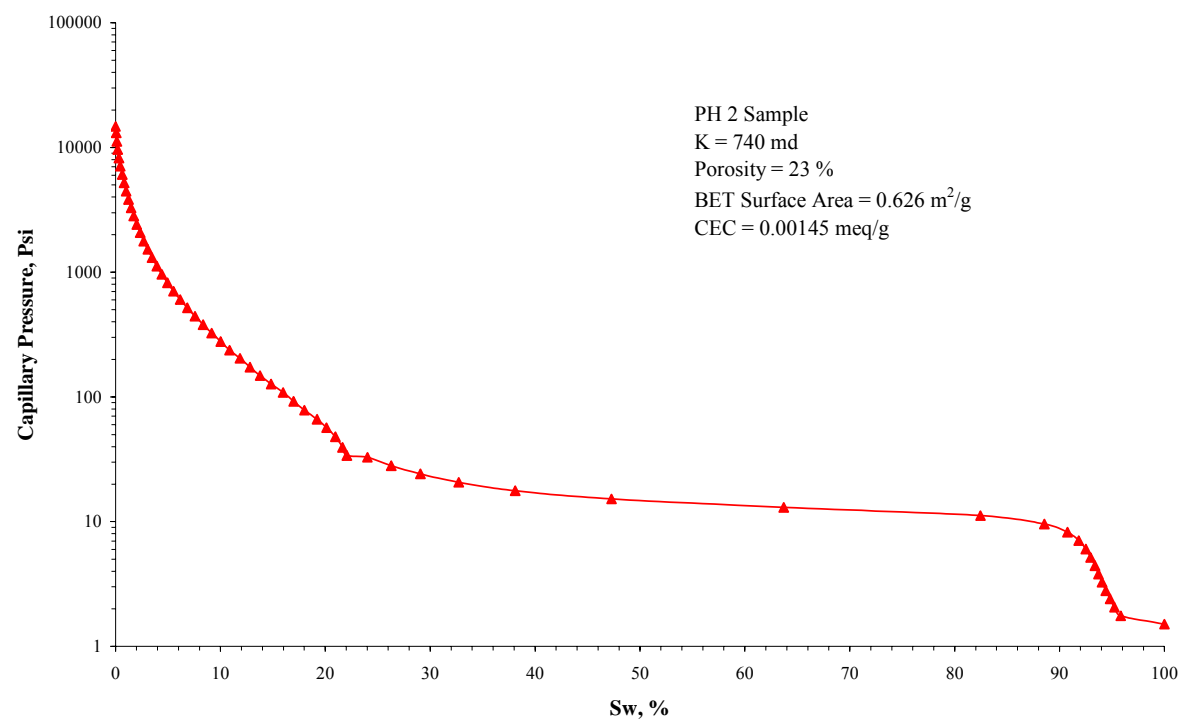

Fig. 1.1 Raw capillary pressure data for PH2 sample.

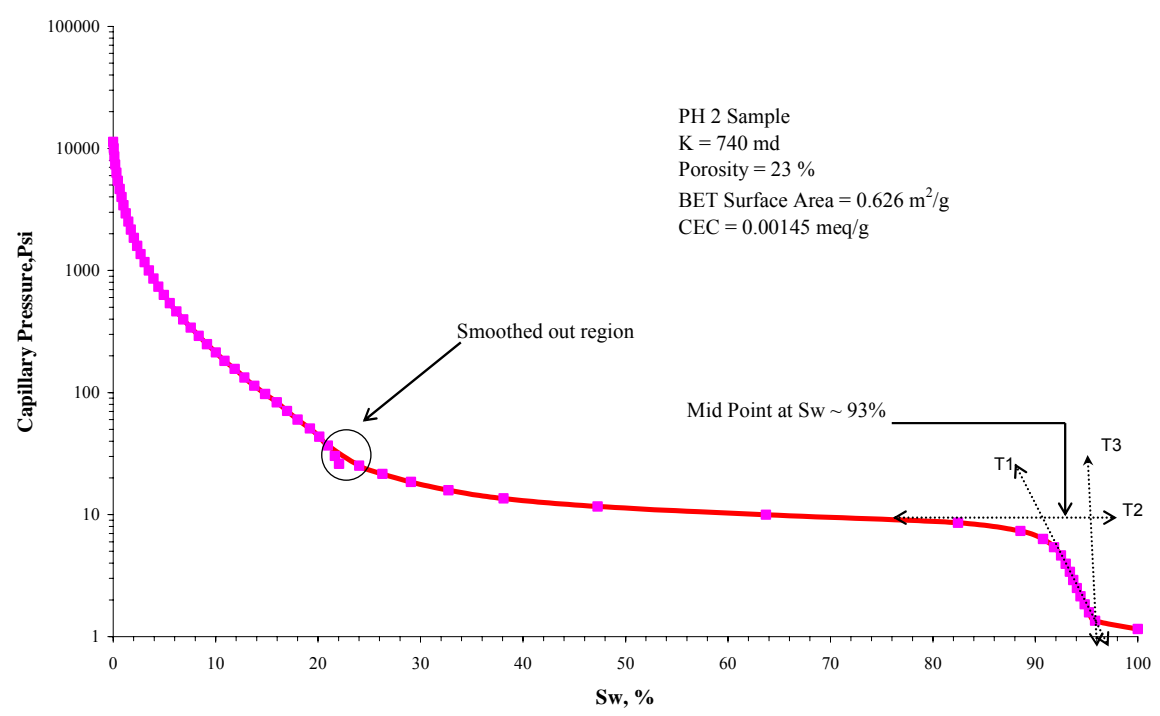

Fig. 1.2. Steps to smooth out the raw mercury injection data. 


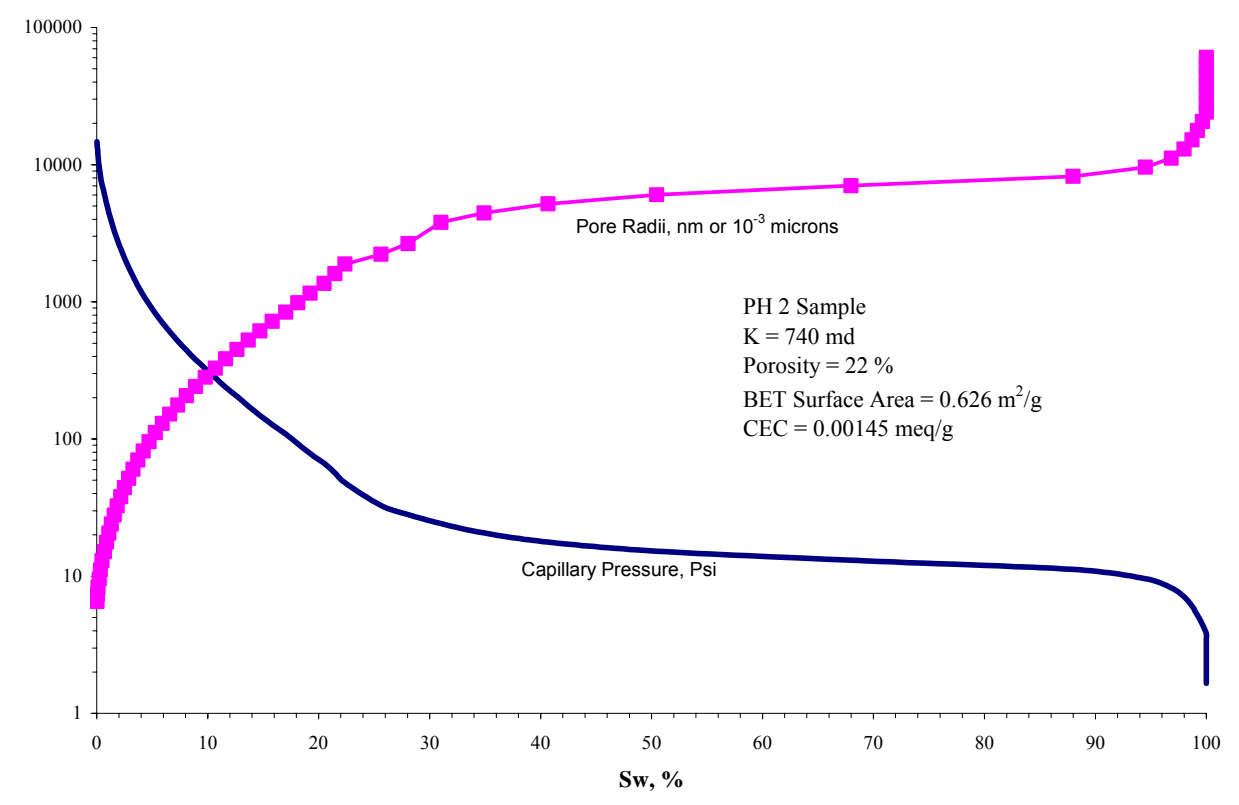

Fig. 1.3. Corrected capillary pressure curve and the radii of investigation for Sandstone (PH2).

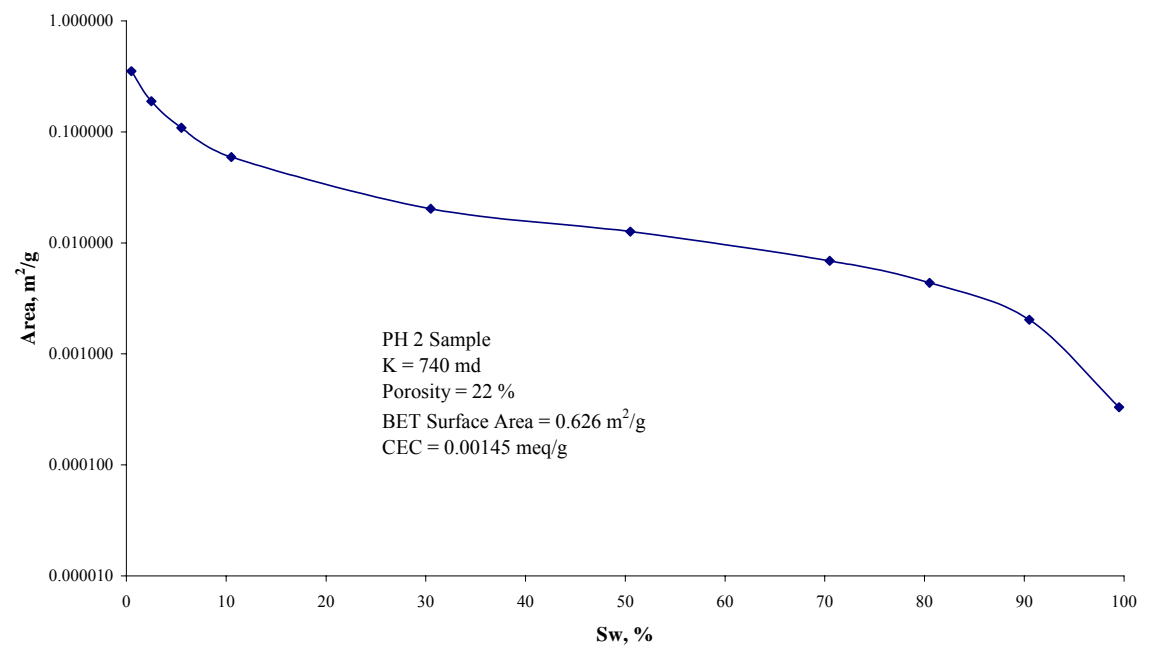

Fig. 1.4. Area obtained by integrating under the capillary pressure curve for the PH2 sample. 


\section{Task 2. Imbibition in simple laboratory and mathematical network models.}

\section{Introduction}

The effect of pore geometry and wetting on capillary displacement pressure has been studied theoretically for a wide variety of pore shapes, using developments of the theory of Mayer and Stowe and Princen, and experimentally for a wide variety of shapes of pore formed from assemblies of rods, plates and spheres (for example, Mason et al., 1983; Mason and Morrow, 1986, 1987, 1991; Ma et al., 1996). Network models of pores have also been studied extensively for example Mason (1971), Sorbie (Dixit et al., 1999), Blunt (1997), and Oren (Oren et al., 1998) but development of network models for spontaneous imbibition lag well behind other advances. One indication of need for a network model is provided by preliminary observations of imbibition by a one end open core. Pressure build up in the nonwetting phase, monitored via a pressure tap, showed a distinct time lag. Such observations indicate that the longstanding assumption (Blair, 1964; Pooladi-Darvish and Firoozabadi, 2000) that capillary pressure immediately rises to a set value, corresponding to residual nonwetting phase saturation at the open core face, needs to be reconsidered. Both this pressure build up and the capillary back-pressure that opposes production of the nonwetting phase at the outflow face must be accounted for in a fundamentally sound network model of counter-current imbibition.

The pressure build up in non-wetting phase, as observed in the imbibition by a one end open core, is closely related to the capillary back-pressure that opposes production of the nonwetting phase at the outflow face. At the pore scale, the complexity of the boundary conditions at the interfaces between fluids and between the fluids and the solid rock matrix means that an exact mathematical description is often difficult to obtain for all but the simplest cases.

Moreover, the fluid motion at the three-phase contact line between moving immiscible fluids and a solid boundary is still not completely understood. Nevertheless it is possible to predict the fluid flow in several idealized pore geometries, such as through cylindrical tubes. In spontaneous imbibition, capillary pressure is considered as the dominant driving force, where the Poiseuille region covers practically the whole of the flow.

As the first step for modeling pore-scale spontaneous imbibition, we presented a simple network model that consists of a number of pore cell units, described as "Hub-and-Spoke Units". Each Unit consists of a sphere (hub) and several connecting cylindrical tubes (spokes) as illustrated in Figure 2.1. Each tube of a Hub-and-Spoke Unit can be classified by one of three possible cases: tube end is open to the network boundary tube; end is a closed end; or end is connected (shared) with another Hub-and-Spoke Unit. Based on the Poiseuille's law, mathematical models have been derived for spontaneous imbibition of water against gas and water against oil in the Hub-and-Spoke Units. Even for a unit cell, it is difficult to find general analytical solutions, except for some simple configurations. Numerical solving schemes and computer codes have, therefore, been developed to simulate the imbibition process in the Huband-Spoke Units. In the preceding quarterly report, detailed numerical solving procedures for spontaneous imbibition of water against oil and water against gas were presented. This quarterly report includes some of the simulation results of spontaneous imbibition in a single Hub-andSpoke Unit. The difference in displacement behavior between water against oil and water against gas is discussed. The simulation was performed on in-house developed $\mathrm{C}++$ codes. 


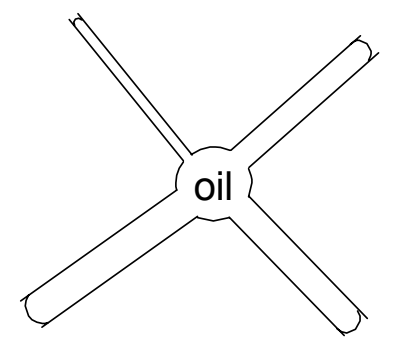

Figure 2.1. A Hub and Spoke Unit with four open-ended tubes.

\section{Experimental}

\section{Spontaneous imbibition of water against oil in Hub-and-Spoke Units}

When gravity force is negligible, capillary pressure becomes the dominant driving force in spontaneous imbibition. As presented in the preceding quarterly report, the spontaneous imbibition of water replacing oil in a Hub-and-Spoke Unit can be described by the Poiseuille's law (Eqs. 2.1 and 2.2), the flow differential equation of Eq. 2.3, and the volume balance equation of Eq. 2.4, in which water and oil are considered as incompressible fluids.

$$
\begin{aligned}
& q_{i w}^{*}=\frac{\pi\left(p_{\text {out }}^{*}-p_{i w}^{*}\right)\left(r_{i}^{*}\right)^{4}}{x_{i}^{*}} b \\
& q_{i o}^{*}=\frac{\pi\left(p_{i o}^{*}-p^{*}(t)\right)\left(r_{i}^{*}\right)^{4}}{L_{i}^{*}-x_{i}^{*}}\left(\frac{\mu_{w}}{\mu_{o}}\right) b \\
& i=1,2, \cdots n \\
& \pi\left(r_{i}^{*}\right)^{2} d x_{i}^{*}=q_{i w}^{*} \frac{d t_{D}}{b} \text { and } q_{i o}^{*}=q_{i w}^{*} \\
& i=1,2, \cdots n \\
& \sum_{i} q_{i o}^{*}=0
\end{aligned}
$$

For generality, we assume that the Unit has $n$ tubes of radii $r_{1} \leq r_{2} \leq \cdots \leq r_{n}$. All tubes are open to the network boundary and the hub has no volume in the following simulated cases. In Eqs. 2.1 to 2.4, parameters $a$ and $b$, and dimensionless variables are defined as:

$$
a=\frac{2 \sigma \cos \theta}{P L}, b=\frac{P}{8 \mu_{w}}
$$

The dimensionless time is defined by

$$
t_{D}=b t
$$

The dimensionless radius, length, and invading position are defined by 


$$
r_{i}^{*}=\frac{r_{i}}{L}, L_{i}^{*}=\frac{L_{i}}{L}, x_{i}^{*}=\frac{x_{i}}{L}, i=1 \sim n
$$

The dimensionless pressure at the center of the Hub-and-Spoke Unit, $p^{*}(t)$, and the dimensionless bulk water pressure outside the Unit, $p_{\text {out }}^{*}$, are defined by

$$
p^{*}(t)=\frac{p(t)}{P}, p_{\text {out }}^{*}=\frac{p_{\text {out }}}{P}
$$

Where $P$ is the unit of pressure measurement; $p(t)$ is the pressure inside the sphere of the Huband-Spoke Unit at any given time $t$. Similarly, the dimensionless capillary pressure in tube $i$ is defined by

$$
p_{c i}^{*}=\frac{p_{c i}}{P}=\frac{a}{r_{i}^{*}}=p_{i o}^{*}-p_{i w}^{*}
$$

In Eq. 2.10, the redefined water and oil flow rates are not dimensionless but have units of 1/t. However, the time unit can be eliminated from the flow differential equation of Eq. 2.3.

$$
q_{i w}^{*}=\frac{q_{i w}}{L^{3}}, q_{i o}^{*}=\frac{q_{i o}}{L^{3}}
$$

Where $L$ is the unit of length measurement; $L_{1}, L_{2}, \cdots, L_{n}$ are the tube lengths; and $x_{1}, x_{2}, \cdots, x_{n}$ are the invading water front positions in tubes at any given time $t$.

For most cases, it will not be easy to find an analytical solution for Eqs. 2.1 to 2.4 but they can be solved numerically. A numerical scheme and its computer code have been developed to simulate water imbibing against oil in a Hub-and-Spoke Unit. The simulation procedure is described in below.

Assuming that we have obtained the advancing/retreating positions of the menisci in tubes, $x_{i}^{* t_{D}}, i=1 \sim n$, at time $t_{D}$, for a given time increment $\Delta t_{D}$, we want to calculate the updated meniscus positions, $x_{i}^{*_{D}+\Delta t_{D}}, i=1 \sim n$. The computation can be summarized by three steps:

(1) Knowing $x_{i}^{* t_{D}}, p_{i w}^{*}\left(t_{D}\right), p_{i o}^{*}\left(t_{D}\right), p^{*}\left(t_{D}\right), i=1 \sim n$

(2) Calculating $x_{i}^{* t_{D}+\Delta t_{D}}, i=1 \sim n$ from Eq. 2.3

(3) Updating the Hub pressure, $p^{*}\left(t_{D}+\Delta t_{D}\right)$, by

$$
p^{*}\left(t_{D}+\Delta t_{D}\right)=\sum_{i} \frac{\left(p_{\text {out }}^{*}+\frac{a}{r_{i}^{*}}\right)\left(r_{i}^{*}\right)^{4}}{L_{i}^{*}+x_{i}^{* t_{D}+\Delta t_{D}}\left(\frac{\mu_{w}}{\mu_{o}}-1\right)} / \sum_{i} \frac{\left(r_{i}^{*}\right)^{4}}{L_{i}^{*}+x_{i}^{* t_{D}+\Delta t_{D}}\left(\frac{\mu_{w}}{\mu_{o}}-1\right)}
$$

(4) Updating the oil phase pressures at menisci, $p_{i o}^{*}\left(t_{D}+\Delta t_{D}\right), i=1 \sim n$, by 


$$
p_{i o}^{*}\left(t_{D}+\Delta t_{D}\right)=\frac{x_{i}^{* t_{D}+\Delta t_{D}} \frac{\mu_{w}}{\mu_{o}}}{L_{i}^{*}+x_{i}^{* t_{D}+\Delta t_{D}}\left(\frac{\mu_{w}}{\mu_{o}}-1\right)} p^{*}\left(t_{D}+\Delta t_{D}\right)+\frac{L_{i}^{*}-x_{i}^{* t_{D}+\Delta t_{D}}}{L_{i}^{*}+x_{i}^{* t_{D}+\Delta t_{D}}\left(\frac{\mu_{w}}{\mu_{o}}-1\right)}\left(p_{\text {out }}^{*}+\frac{a}{r_{i}^{*}}\right)
$$

(5) Updating the water phase pressures at menisci, $p_{i w}^{*}\left(t_{D}+\Delta t_{D}\right), i=1 \sim n$, from Eq. 2.9

The above five steps for each time increment are repeated until the spontaneous imbibition process is completed. At the end of imbibition, oil is trapped only in the tubes with the largest radii in the Unit.

\section{Spontaneous imbibition of water replacing gas in Hub-and-Spoke Units}

The same configuration of Hub-and-Spoke Unit was used to simulate the spontaneous imbibition of water against gas. Here, the Unit is initially saturated with gas instead of oil and the gas viscosity is negligible. The numerical solving scheme for water-gas spontaneous imbibition was initially introduced in the last quarterly report. For consistency, derivation of the mathematical model derivation and its numerical solving scheme are presented.

When the gas inside the Unit is compressed by invading water, the gas obeys Boyle's Law: for a given mass, at constant temperature, the pressure times the volume is a constant.

$$
p V=m R T
$$

where $p$ is the gas pressure, $V$ is the gas volume, $T$ is temperature, $m$ is the number of moles, and $R$ is the gas constant. For air, $R=0.286 \mathrm{kj} / \mathrm{kg} / \mathrm{K}^{\mathrm{o}}$.

Let $V^{0}$ and $V^{t}$ represent the initial gas volume and the gas volume at time $t$. We have

$$
V^{0}=\sum_{i} \pi L_{i} r_{i}^{2}, \quad V^{t}=\sum_{i} \pi\left(L_{i}-x_{i}^{t}\right) r_{i}^{2}
$$

or in the dimensionless format

$$
\begin{aligned}
& V^{* 0}=\frac{V^{0}}{L^{3}}=\sum_{i} \pi L_{i}^{*} r_{i}^{* 2}, \quad V^{* t}=\frac{V^{t}}{L^{3}}=\sum_{i} \pi\left(L_{i}^{*}-x_{i}^{* t}\right) r_{i}^{* 2} \\
& i=1,2, \cdots n
\end{aligned}
$$

According to Boyle's Law of Eq. 2.22, we obtain

$$
p^{*}\left(t_{D}\right)=\frac{p^{* 0} V^{* 0}}{V^{* t_{D}}}
$$

In addition, if the gas viscosity is neglected, gas pressure is the same everywhere inside the Hub and Spoke Unit. For the trapped gas, Poiseuille's law (Eq. 2.1) gives, 


$$
\begin{aligned}
& q_{i w}^{*}=\frac{\pi\left(p_{\text {out }}^{*}-\left(p^{*}\left(t_{D}\right)-p_{c i}^{*}\right)\right) r_{i}^{* 4}}{8 \mu_{w} x_{i}^{t}} b \\
& i=1,2, \cdots n
\end{aligned}
$$

Eqs. 2.3, 2.15, 2.16, and 2.17 form the mathematical model for the water-gas spontaneous imbibition in a Hub-and-Spoke Unit. A numerical scheme, described below, has been developed to solve the proposed mathematical model.

Assuming that we have obtained the advancing/retreating positions in each tube, $x_{i}^{* t_{D}}, i=1 \sim n$, at time $t_{D}$, for a given time increment $\Delta t_{D}$, we want to calculate $x_{i}^{*{ }^{*} t_{D}+\Delta t_{D}}, i=1 \sim n$. The computation can be summarized by three steps:

(1) Knowing $x_{i}^{* t_{D}}, p^{*}\left(t_{D}\right), i=1 \sim n$

(2) Calculating $x_{i}^{* t_{D}+\Delta t_{D}}, i=1 \sim n$ from Eqs. 2.3 and 2.17, precisely,

If $\left(p_{\text {out }}^{*}-\left(p^{*}\left(t_{D}\right)-p_{c i}^{*}\right)\right) \geq 0$ then $x_{i}^{* t_{D}+\Delta t_{D}}=\sqrt{\left(x_{i}^{* t_{D}}\right)^{2}+2\left(p_{\text {out }}^{*}-p^{*}\left(t_{D}\right)+p_{c i}^{*}\right)\left(r_{i}^{*}\right)^{2} \Delta t_{D}}$ If $\left(p_{\text {out }}^{*}-\left(p^{*}\left(t_{D}\right)-p_{c i}^{*}\right)\right)<0$ then $x_{i}^{* t_{D}+\Delta t_{D}}=2 x_{i}^{* t_{D}}-\sqrt{\left(x_{i}^{* t_{D}}\right)^{2}-2\left(p_{\text {out }}^{*}-p^{*}\left(t_{D}\right)+p_{c i}^{*}\right)\left(r_{i}^{*}\right)^{2} \Delta t_{D}}$

(3) Updating $p^{*}\left(t_{D}\right)$ from Eqs. 2.15 and 2.16.

The above three steps are repeated for each time increment until spontaneous imbibition process is complete. When imbibition begins, water invades every tube as gas is being compressed. Once the compression phase is finished, the gas bubble inside the Unit will expand and be pushed out from the largest tube(s), by capillary pressure acting in the smaller tubes. At the end of imbibition, gas is trapped only in the largest tube(s) of the Unit. Some of the simulation results are presented in the next section.

\section{Results and Discussion}

Coded by $\mathrm{C}++$, console applications have been developed for simulating the spontaneous imbibition in the Hub-and-Spoke Units. The two codes, named SpiderWO and SpiderGas, are used to simulate the imbibitions of water against oil and gas, respectively. Examples of the formatted input files for SpiderWO and SpiderGas are shown in Figure 2.2 and 2.3. For a simulation run, the input file defines the configuration of a Hub-and-Spoke Unit, initial pressures, total simulation time, time step and printing time step. With various parameter settings, the time-lag phenomena at different pore scales can be investigated by the simulation. Some preliminary results are discussed below. 
//This is a formatted input file for SpiderWO (water/oil). Please do not insert or delete any lines.

//Dimensionless Units are used in computation

//Using a space to separate parameters if they are entered in one line.

\#\# Begin of data input: unit length - $\mathrm{cm}$, time - second

//--- Initial outside (water) pressure (in psi) \& the scale-pressure (in dynes/cm2)

14.71 .0

//--- a \& b (two parameters), Lambda: water/oil viscosity ratio ---

60.012 .51 .5

//--- TD \& delta tD, print_step (i.e. printing once for every print_step of tD) ---

1000.00210

//--- Tube Configuration ---

//Number of tubes

5

//--- Tube Length ---

11111

//--- Tube Radius ---

0.0050 .0070 .0080 .0090 .01

\#\# End of data input

Figure 2.2. Example of the formatted input file for the SpiderWO console application.

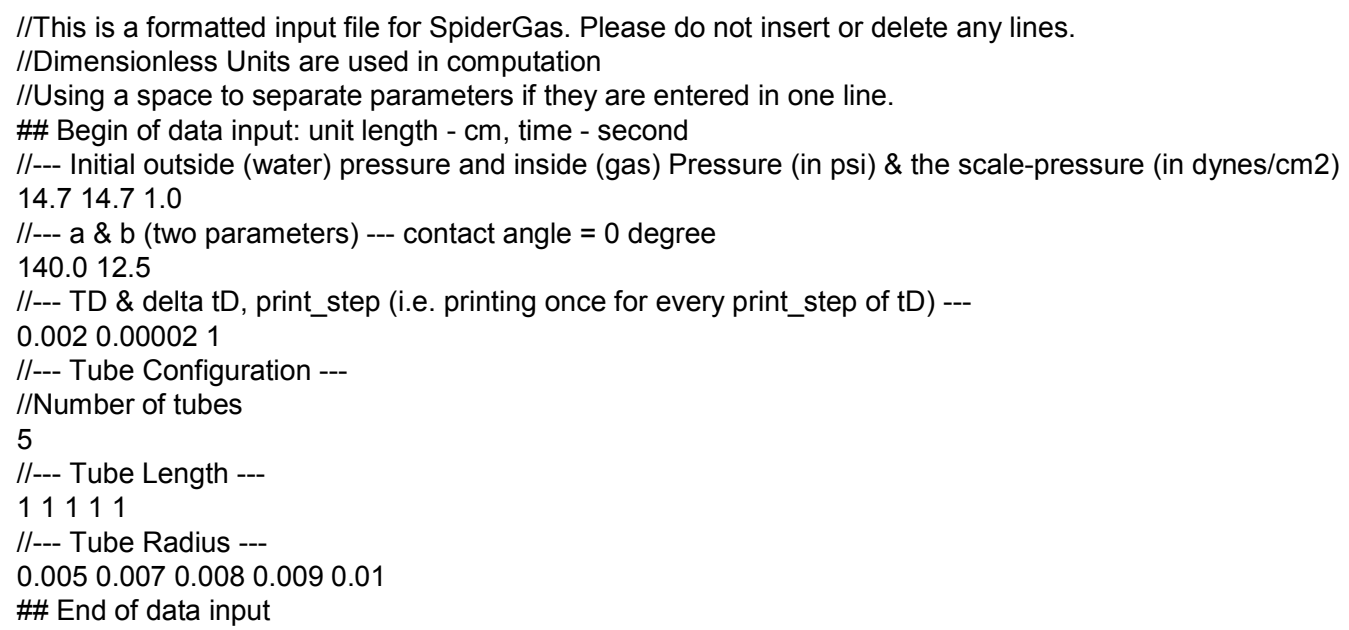

Figure 2.3. Example of the formatted input file for the SpiderGas console application.

\section{Imbibition simulation of water against gas}

As observed in laboratory experiments on core samples, the gas in core samples is first compressed at the beginning of a spontaneous imbibition process until gas breaks through from the pores with the largest size in diameter at the outflow face. When the gas inside the Unit is compressed by invading water, the gas obeys the Boyle's Law and, therefore, an increase in gas pressure is observed. In this first attempt, a single Hub-and-Spoke Unit was used to simulate the, so called, time-lag that precedes gas production. The configuration of the Unit is given in Table 2.1. The fluid properties, initial condition, and other parameter settings used in the simulation are listed in Table 2.2. The wettability effect was simulated by using different contact angles. 
Table 2.1. The configuration of the simulated Hub-and-Spoke unit

\begin{tabular}{|c|cc|}
\hline Hub Volume & \multicolumn{2}{|c|}{0} \\
\hline Spoke & Radius, $\boldsymbol{\mu m}$ & Length, $\mathbf{~ c m}$ \\
\hline Spoke 1 & 50 & 1 \\
Spoke 2 & 70 & 1 \\
Spoke 3 & 80 & 1 \\
Spoke 4 & 90 & 1 \\
Spoke 5 & 100 & 1 \\
\hline
\end{tabular}

Table 2.2. Fluid properties and parameters used in water replacing gas

\begin{tabular}{|l|l|}
\hline Initial gas pressure inside the Unit, psi & 14.7 \\
Bulk water pressure outside the Unit, psi & 14.7 \\
Water viscosity, $\mu_{\mathrm{w}}, \mathrm{cp}$ & 1 \\
Water-gas interfacial tension, $\sigma, \mathrm{dyn} / \mathrm{cm}$ & 70 \\
Contact angle, degree & $0,15,30,45$ \\
$a$ & $140,135,121,99$ \\
$b, 1 /$ second & 12.5 \\
\hline
\end{tabular}

Note that, in Table 2.1, no hub volume was assumed in the simulations. The assumption was used, as a demonstration, for simplifying the computation only. In a network model that represents a certain porous medium, the total volume of the Hubs and Spokes in a cubic unit should be equivalent to the porosity of the given porous medium.

As shown in Figs. 2.4 and 2.5, the contact angle, via capillary pressure, has a notable effect on the initial gas compression and the gas recovery rate. For a larger contact angle, therefore smaller capillary pressures, it takes less induction time for compressing the gas, Fig. 2.4, but more time for recovering the gas from the Unit, Fig. 2.5. During the compression phase, water-gas menisci in all tubes first move toward to the Hub as the gas pressure builds up, Figs. 2.4 and 2.6. After reaching pressure equilibrium, the gas bubble inside the Unit begins to move toward the largest tube of the Unit, Fig. 2.6. As the gas bubble expands again and its pressure drops accordingly, Fig. 2.4, before the gas breakthrough from the Unit. Figure 2.7 shows the dimensionless water flow rate in each spoke before gas breakthrough. The dimensionless flow rate is defined by Eq. 2.18, where $q^{*}$ is the flow rate given by Eq. 2.10 or 2.17 .

$$
q_{D}=q^{*} / b
$$

As shown in Fig. 2.7, the water outflow rate in the tube of $100 \mu \mathrm{m}$ radius increases rapidly when its water-gas meniscus approaches the tube's outlet end. Because the gas viscosity is neglected and therefore the gas pressure is the same everywhere in the Unit, the gas bubble can only flows out from the tubes with the largest radius. This flow pattern will not be always true in imbibition of water against oil, which will be discussed in the next section. 


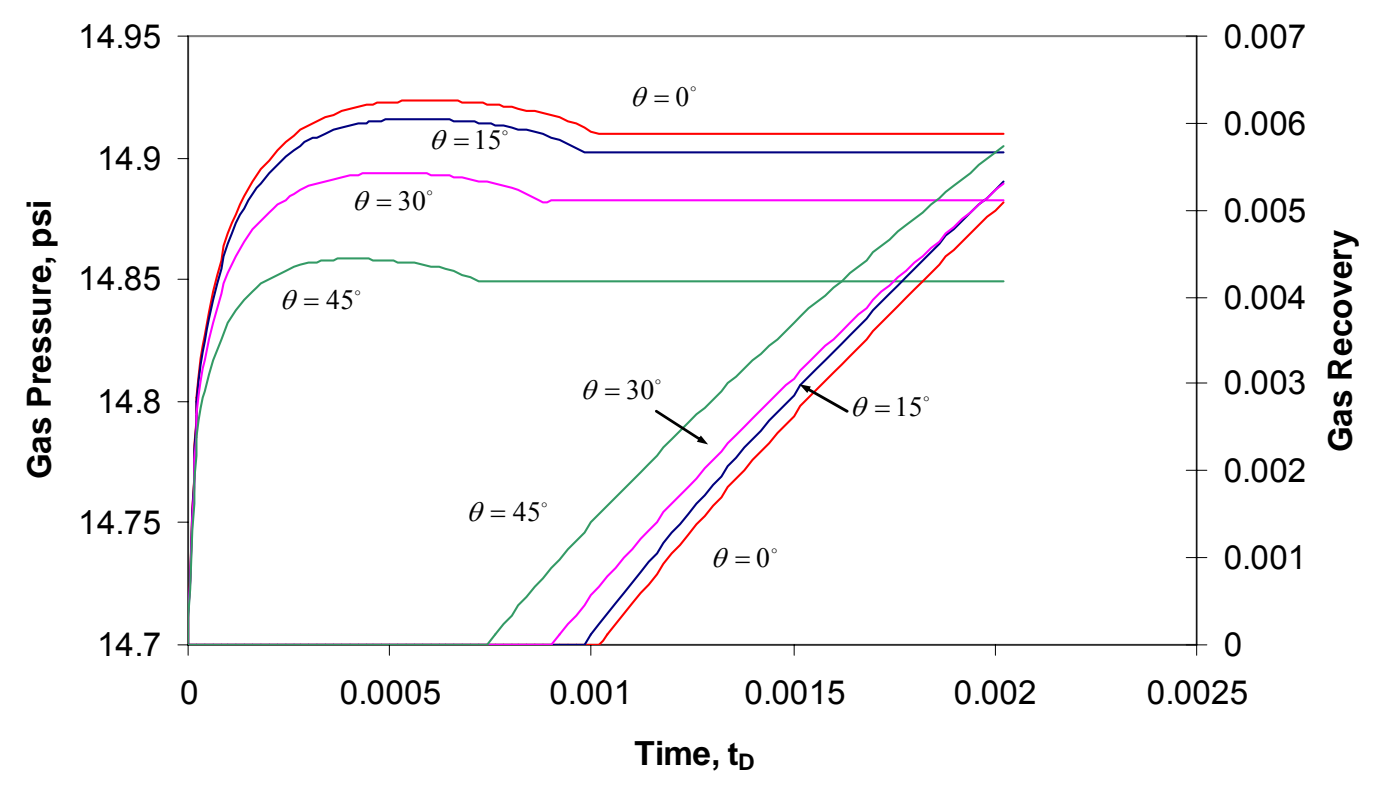

Figure 2.4. The effect of contact angle on the initial gas compression and the recovery induction time.

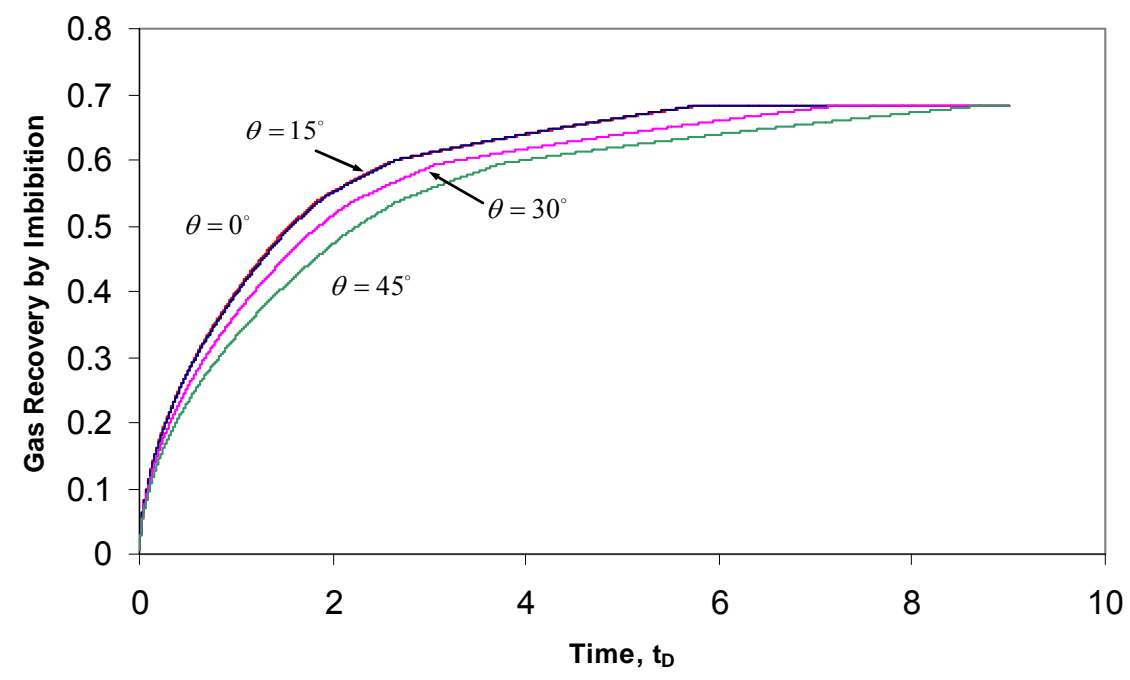

Figure 2.5. The gas recovery rates simulated by using different contact angles 


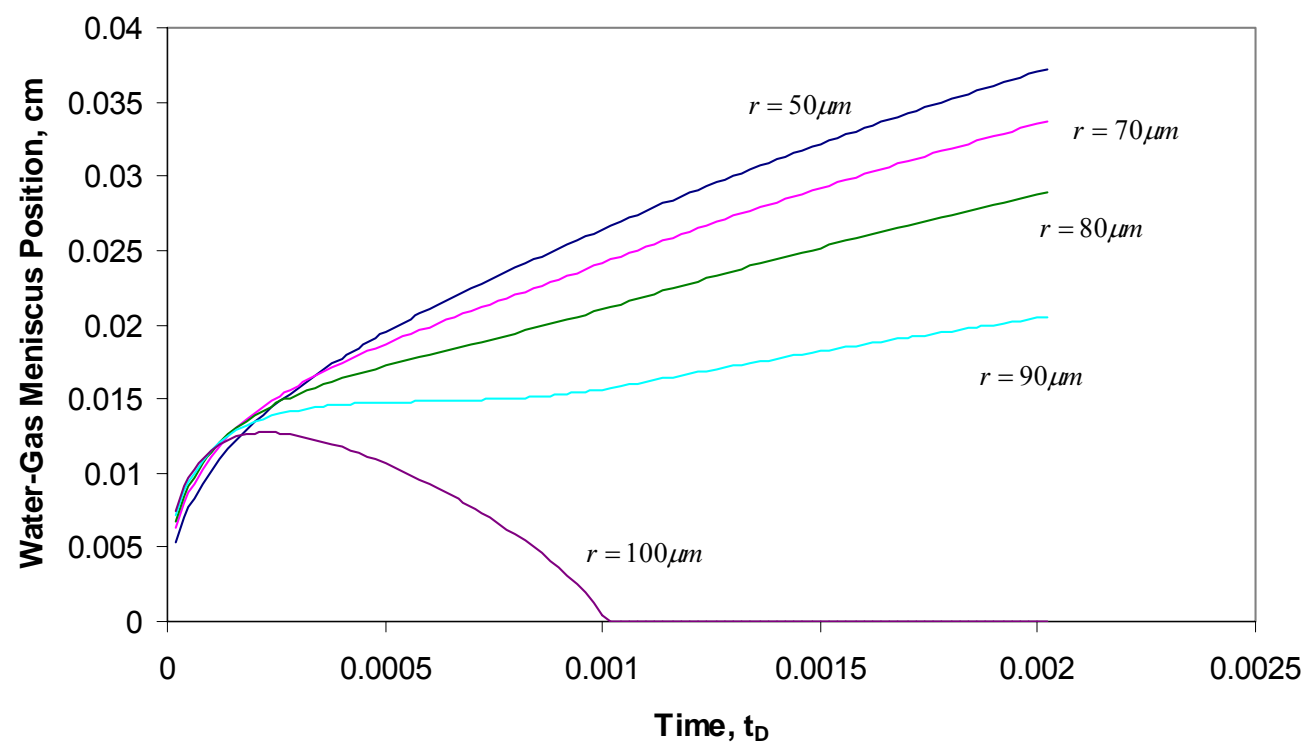

Figure 2.6. The advancing/receding behavior of water-gas menisci in spokes during the initial gas compression phase of spontaneous imbibition. (contact angle $=0^{\circ}$ )

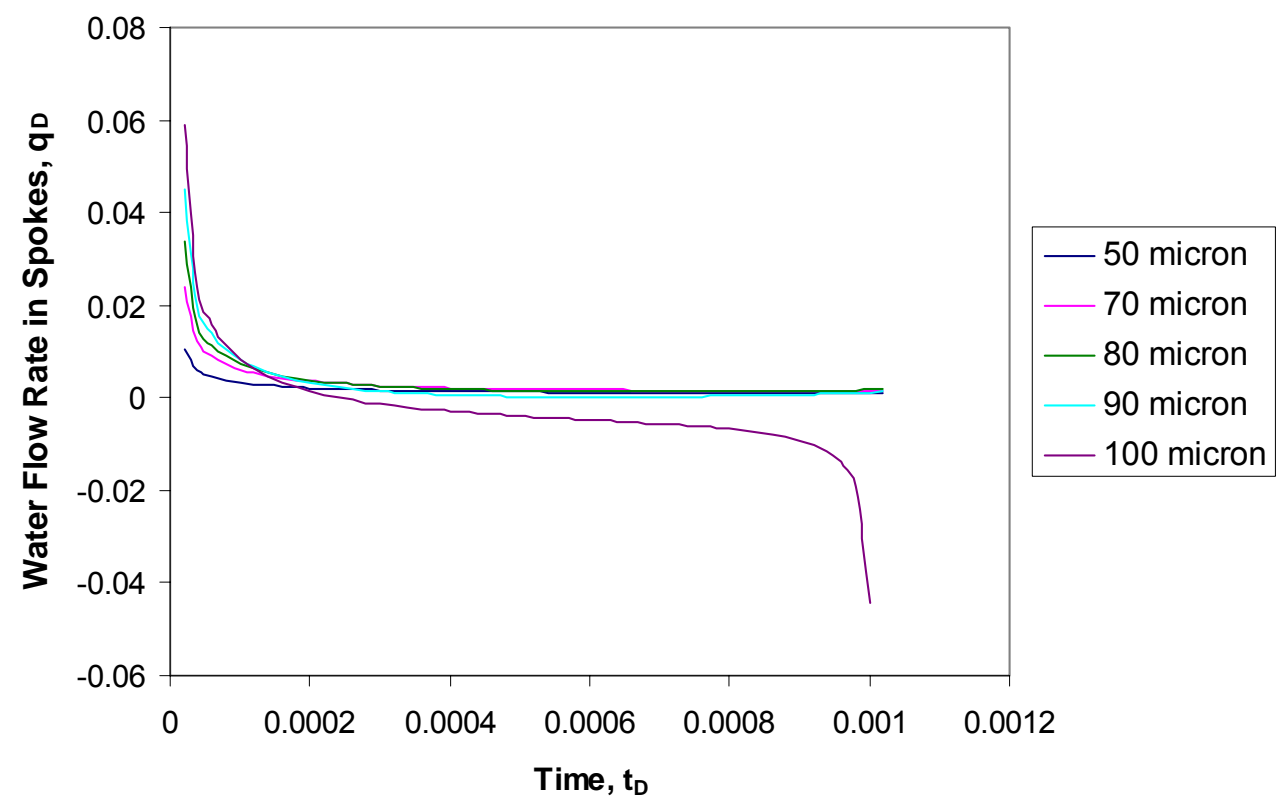

Figure 2.7. The water flow rates in spokes before gas breakthrough from the spoke with the largest radius. (contact angle $=0^{\circ}$ ) 


\section{Imbibition simulation of water against oil}

The time-lag phenomena during pressure build up in the non-wetting phase in the imbibition of water against oil is not as significant as that in water against gas, because water and oil have much smaller compressibility in comparison to gas. It is assumed, here, that both oil and water are incompressible. For imbibition of water against oil, the rate of oil (non-wetting phase) recovery by spontaneous imbibition is strongly correlated with the viscosity ratio of water (wetting phase) to oil (non-wetting phase). The same configuration of Hub-and-Spoke Unit, Table 2.1, was used to simulate water-oil imbibition. However, the focus is now on the effect of water-oil viscosity ratio. The simulated water-oil viscosities are listed in Table 2.3 along with other fluid properties and parameter settings. For all simulated cases a fixed water viscosity of 1 $\mathrm{cp}$ is used while the oil viscosity varies.

Figure 2.8 shows the effect of oil-water viscosity ratio on the Hub pressure. When a meniscus in any tube reaches the Hub, a drop in Hub pressure is observed, a process of snap off. Between any two snap off processes, the dynamic pressure in the Hub is controlled by the viscosity ratio. For higher oil-water viscosity ratio, it takes more time to complete the imbibition process, Fig. 2.9, which is consistent with the core imbibition results. At the end of imbibition, oil is trapped only in the tube with the largest radius in the Unit. Figure 2.10 and 2.11 show the movements of oil-water menisci in spokes and the dimensionless spoke flow rates, respectively, simulated with an oil-water viscosity ratio of 2 . Similar results with viscosity ratios of 1 and 0.67 are given in Figs. $2.12 \sim 2.15$. In contrast to water-gas imbibition, the flow pattern in water-oil imbibition is much more complicated. In addition to the pressure fluctuation caused by snap off, the flow direction in a tube can also be alternated during the imbibition process, as happened to the tube that has the second largest radius, $90 \mu \mathrm{m}$, in the simulated cases. In the next step, we will simulate for a broader configurations using the current codes. To the second phase of the project, the model will be extended to mixed wettability and non-cylindrical tubes.

Table 2.3. Fluid properties and parameters used in water replacing oil

\begin{tabular}{|l|l|}
\hline Initial oil pressure inside the Unit, psi & 14.7 \\
Bulk water pressure outside the Unit, psi & 14.7 \\
Water viscosity, $\mu_{\mathrm{w}}, \mathrm{cp}$ & 1 \\
Oil viscosity, $\mu_{\mathrm{o}}, \mathrm{cp}$ & $0.667,1,2$ \\
Water-oil interfacial tension, $\sigma, \mathrm{dyn} / \mathrm{cm}$ & 30 \\
Contact angle, degree & 0 \\
$a$ & 60 \\
$b, 1 /$ second & 12.5 \\
\hline
\end{tabular}




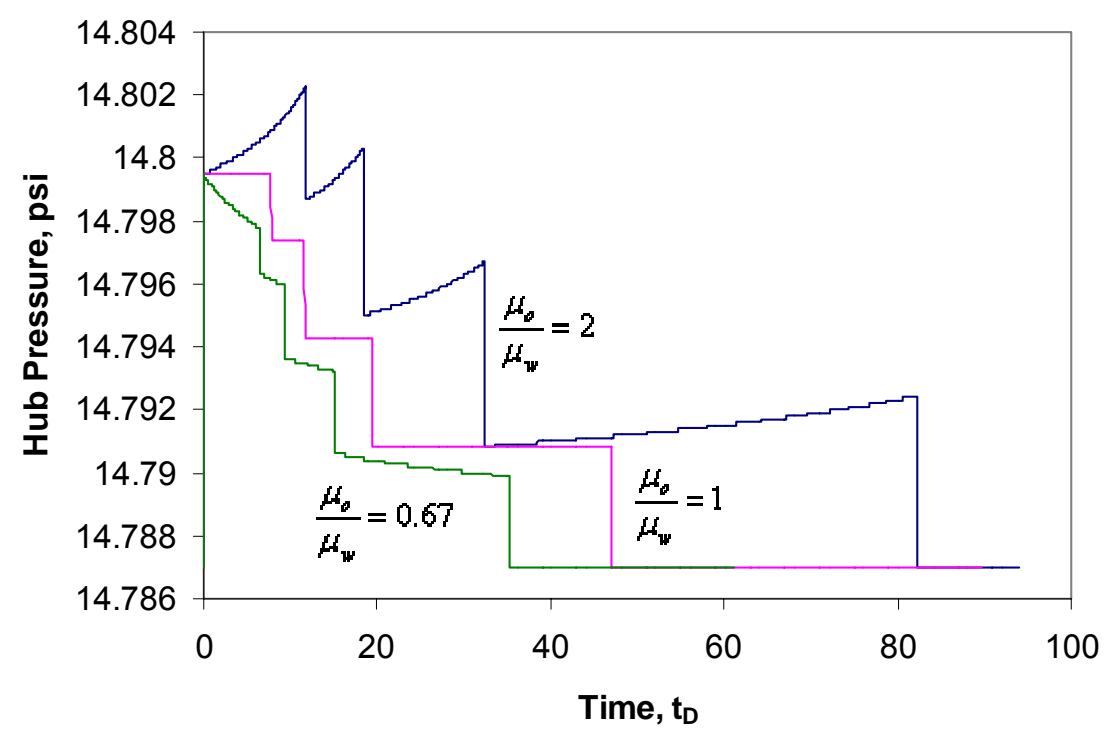

Figure 2.8. The effect of oil-water viscosity ratio on the Hub pressure, where a water viscosity of $1 \mathrm{cp}$ was used for all cases.

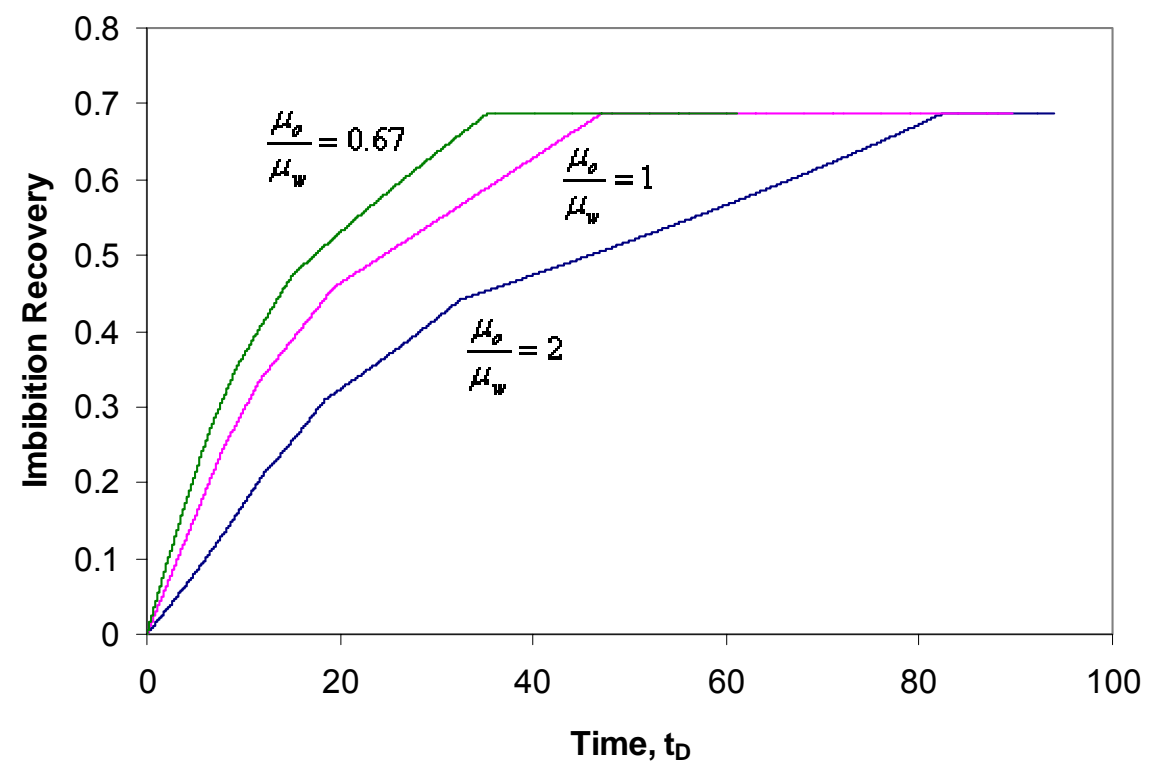

Figure 2.9. The effect of oil-water viscosity ratio on the oil recovery by spontaneous imbibition. (water viscosity of $1 \mathrm{cp}$ for all cases). 


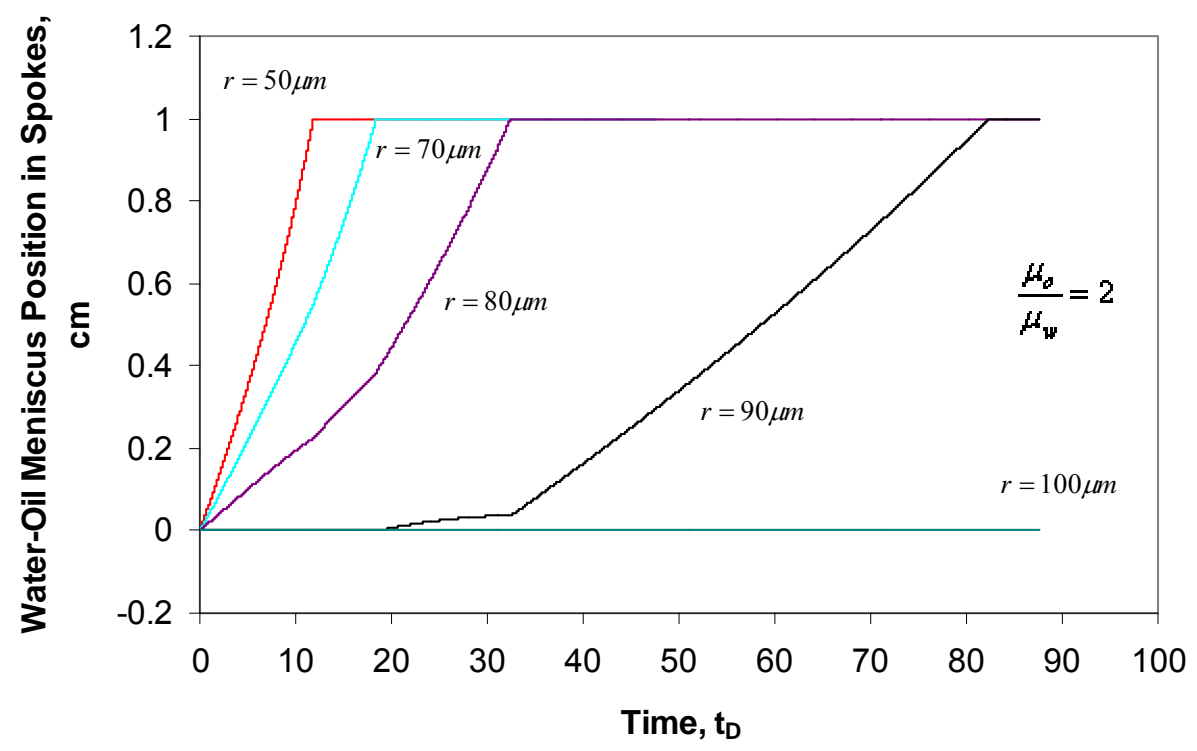

Figure 2.10. Advancing of oil-water menisci in spokes simulated with an oil-water viscosity ratio of 2.

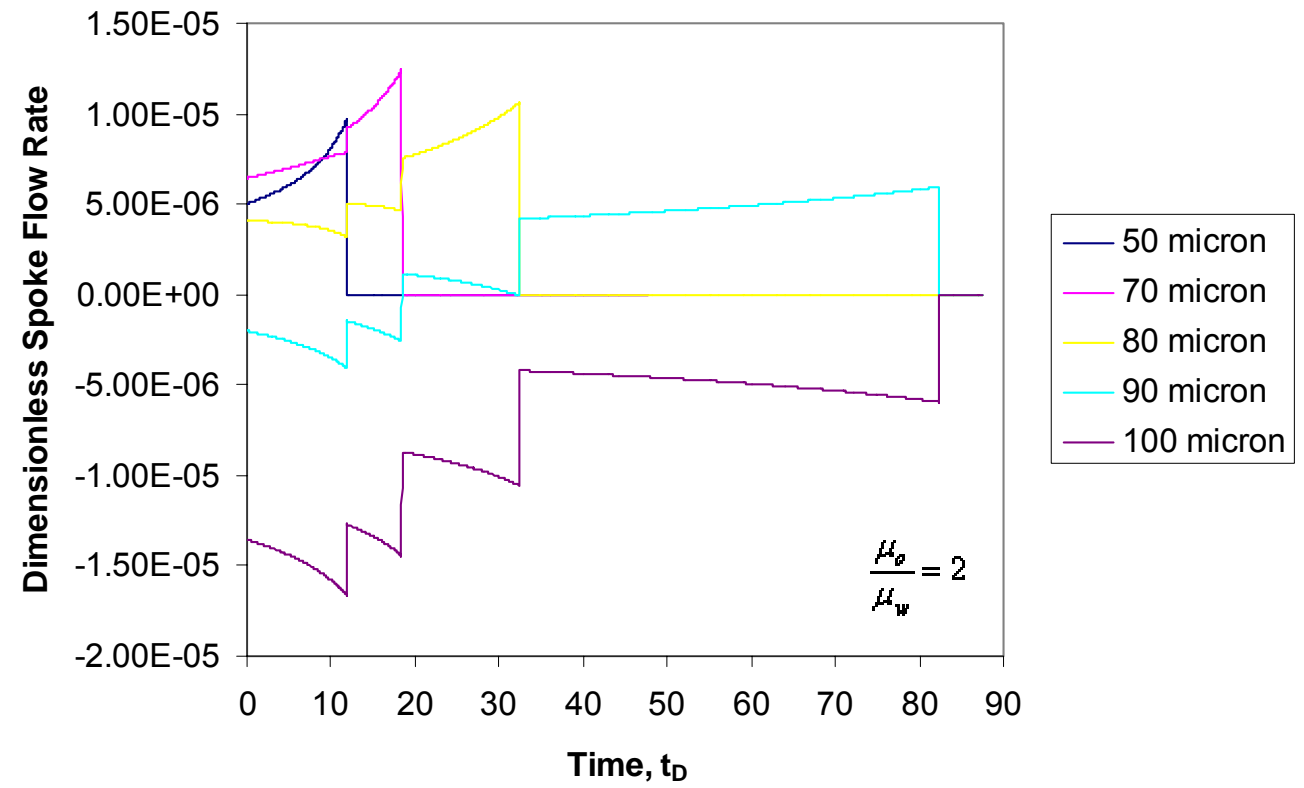

Figure 2.11. Dimensionless spoke flow rates simulated with an oil-water viscosity ratio of 2. 


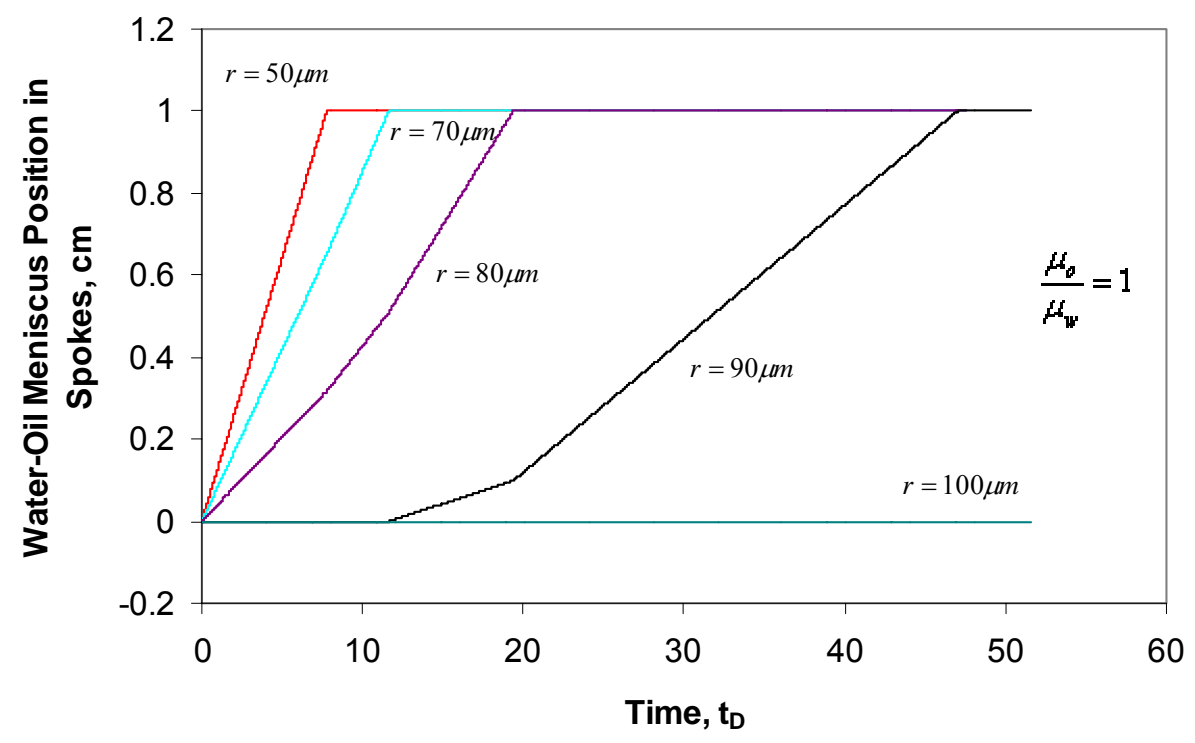

Figure 2.12. Advancing of oil-water menisci in spokes simulated with an oil-water viscosity ratio of 1.

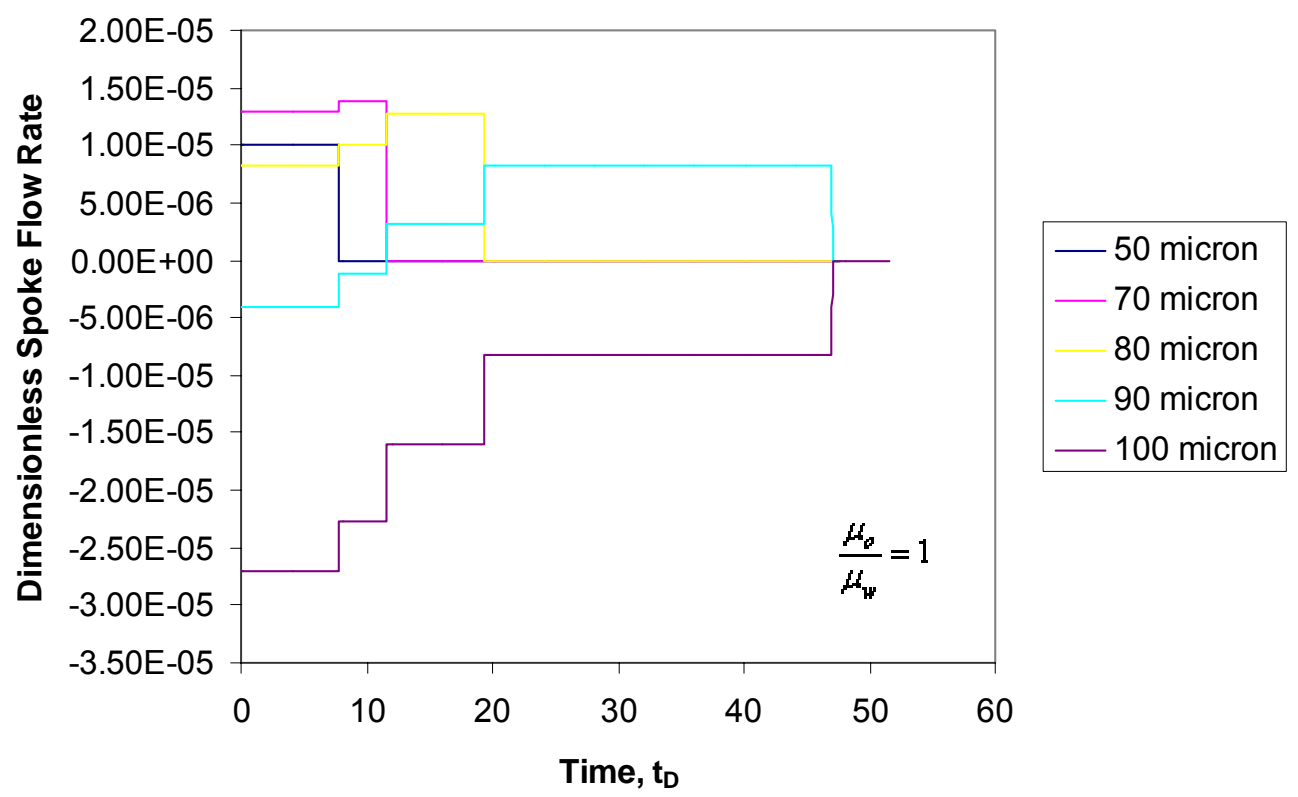

Figure 2.13. Dimensionless spoke flow rates simulated with an oil-water viscosity ratio of 1 . 


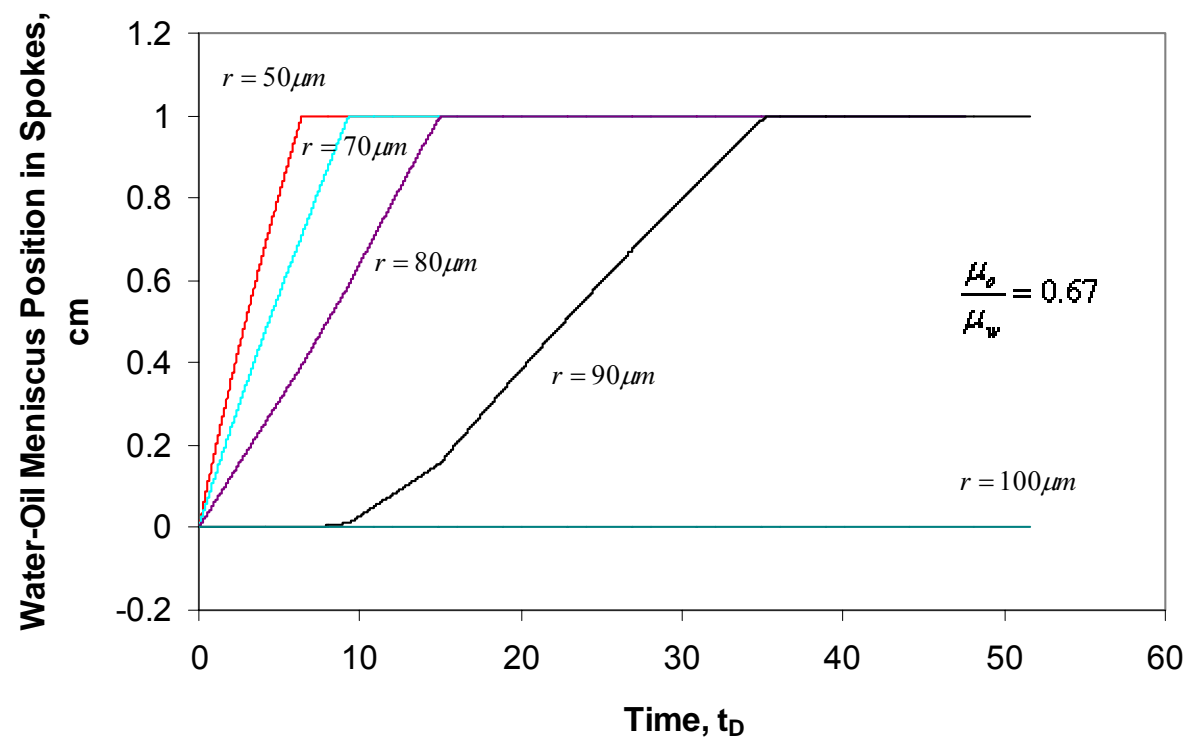

Figure 2.14. Advancing of oil-water menisci in spokes simulated with an oil-water viscosity ratio of 0.67 .

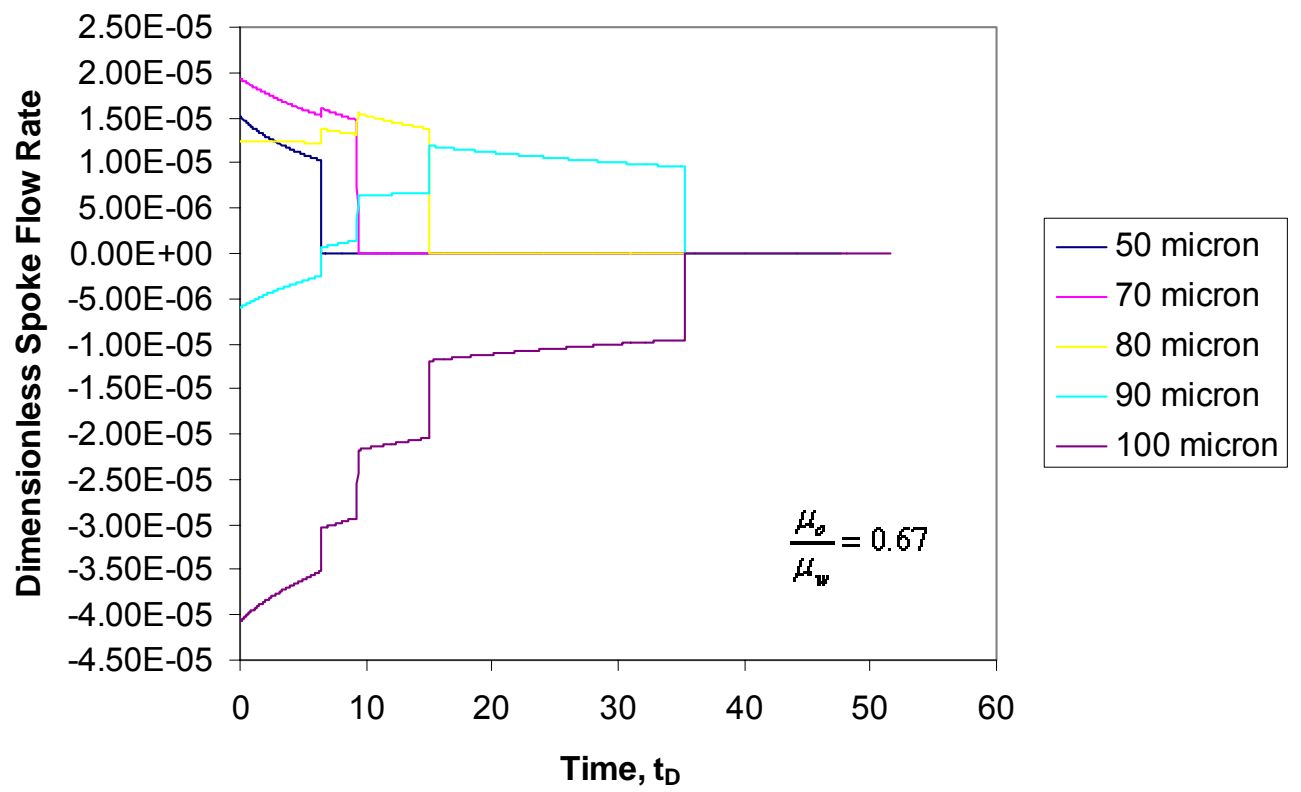

Figure 2.15. Dimensionless spoke flow rates simulated with an oil-water viscosity ratio of 0.67 . 
Task 3. Novel observations on fluid pressures during imbibition and the mechanism of non-wetting phase production at the imbibition face.

\section{Introduction}

Simulation of nonwetting phase production

The mathematical model used to develop previously reported simulations is presented below together with the functions used to describe variation in capillary pressure and relative permeabilities.

\section{Experimental}

Construction of $P_{c} / \sigma$ and $k_{r w} / K_{w e}$ and $k_{r n w} / K_{n w e} \underline{\text { functions }}$

For the measured relative permeability curves of Berea sandstone [Fulcher et al. 1985], the relative permeabilities were modeled by,

$k_{r w} / K_{w e}=\left[\left(k_{r n w 0}-k_{r n w f}\right)\left(\frac{1-S_{w o}-S_{w}}{1-S_{w o}-S_{w f}}\right)^{N_{w}}+k_{r n w f}\right]$

and

$k_{n r w} / K_{n w e}=\left[\left(k_{r w f}-k_{r w 0}\right)\left(\frac{S_{w f}-S_{w}}{1-S_{w 0}-S_{w f}}\right)^{N_{n w}}+k_{r w 0}\right]$

where $K_{w e}$ and $K_{n w e}$ are the effective permeabilities for the WP and the NWP respectively, $S_{w f}$ is the $S_{w}$ at the front, $k_{r w f}$ is the relative permeability at the front, and $k_{r w 0}$ is the relative permeability at the open face.

$K_{w}$ (brine) is 0.55 times $K_{\mathrm{g}}$ (gas) (Chatzis and Morrow, 1984). The permeability to oil, $K_{o}$ was 0.8 times $K$ for air $\left(K_{a i r}\right)$ (Fig.3.1). The relative permeability for brine, $K_{w}$ at the residual oil saturation $\left(S_{o r}\right), K_{w}\left(S_{o r}\right)$ is 0.0458 . In simulation we adopted 0.04 as the value of the brine relative permeability at the open face and 0.0727 for oil. 


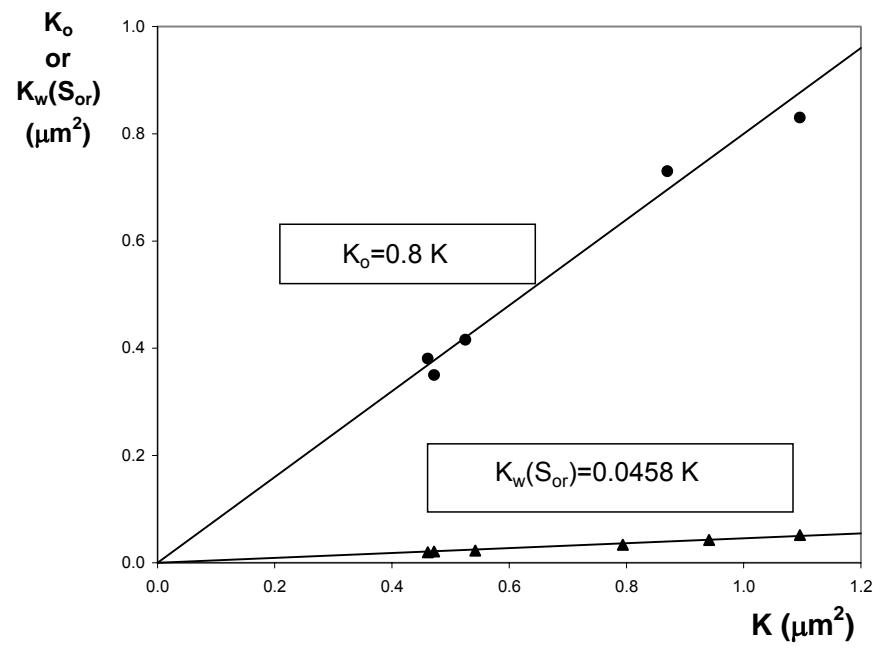

Fig. 3.1 $K_{o}$ versus $K$ (gas permeability) and $K_{w}\left(\mathrm{~S}_{\text {or }}\right)$ versus $K$

From the measured imbibition capillary pressure curve of Berea sandstone [Wardlaw and Taylor, 1976], the capillary pressure function was also assumed to be parabolic in form for COUCSI

$$
\frac{P_{c}}{\sigma}=\frac{\sigma_{o-w}}{\sigma_{a-o}}\left(\frac{P_{c f}}{\sigma}-\frac{P_{c 0}}{\sigma}\right)\left(\frac{1-S_{w o}-S_{w}}{1-S_{w o}-S_{w f}}\right)^{N_{p c}}+\frac{P_{c 0}}{\sigma}
$$

The variables of the curves used for each core are listed in Table 2. Results for Core H8A are plotted in Fig. 3.2. 


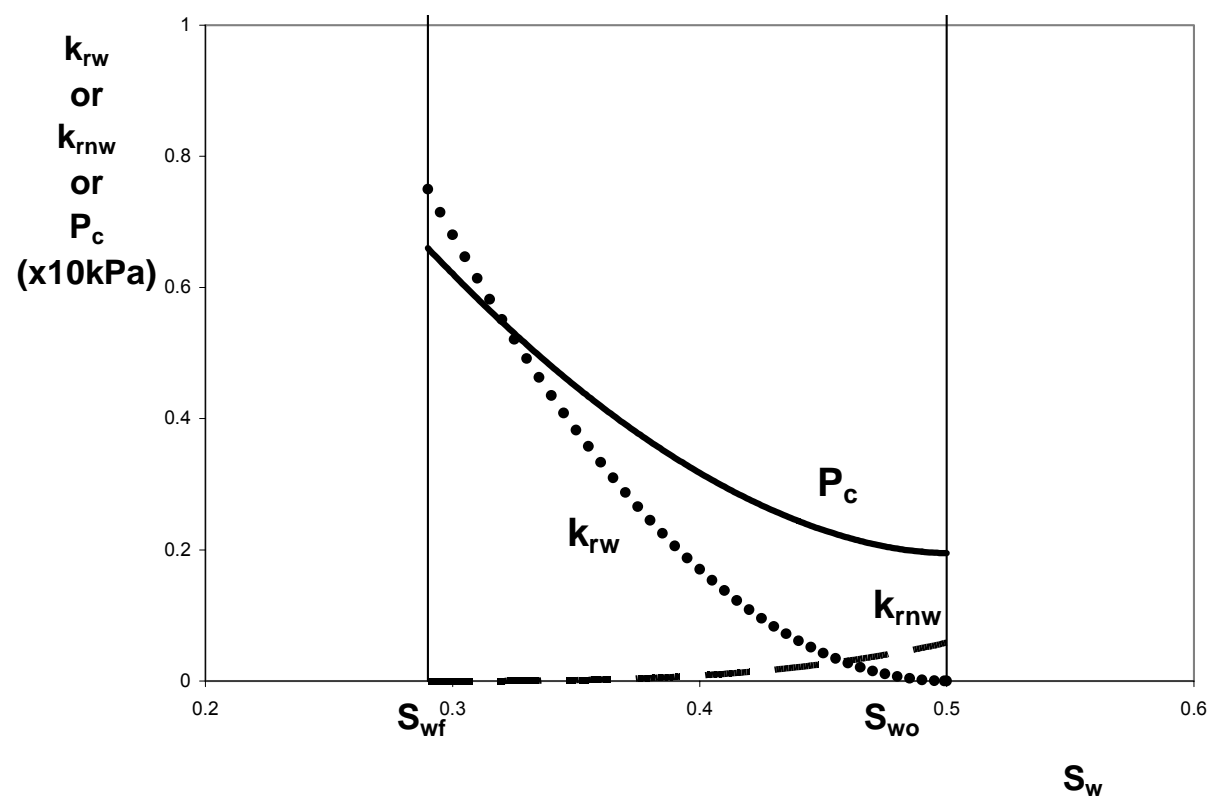

Fig. 3.2 Relative permeabilities and capillary pressure for Core H8A change ks

\section{Results and Discussion}

Even with the described constraint on the possible sets of $k_{r w}, k_{r n w}$ and $P_{c}$ curve shapes, simulations could be performed which provided a close match with the three measured parameters of a COUCSI experiment. Furthermore, a single set of $k_{r w}, k_{r n w}$ and $P_{c}$ curves can be obtained for both water/oil and oil/air COUCSI experiments with duplicate core samples. A quick search for the appropriate set of $k_{r w}, k_{r n w}$ and $P_{c}$ curves was performed using the following steps.

a. Fix $S_{w 0}$ at the experiment value, fix $k_{r w}$ at $S_{w 0}$ as $10^{-10}$ (using $10^{-10}$ instead of zero to avoid occurrence of a zero-divide), fix $k_{r n w} / K_{e}$ at $S_{w f}$ at an arbitrary value, say 0.8 and $k_{r w} / K_{e}$ at $S_{w 0}$ as $0.04 / K_{e}$. Fix $N_{n w}$ at an arbitrary value, say 2 (In the oil/air case the resistance to flow of the NWP is very small). Find appropriate $N_{p c}$ and $N_{w}$ and $S_{w f}$ values to match the oil/air experiments. (The simulated final saturation can be lower).

b. Increase the simulation $S_{w 0}$ to match the final recovery (This procedure will adjust $k_{r n w}$ at the experimental $S_{w 0}$ to be finite.).

c. Change $N_{w}$ and $P_{c b}$ so that the simulation curves match the water/oil performances ( $P_{n w}$ at the dead end during the post contact period can be different from $P_{\text {end }}$ after WP has arrived at the dead end.)

d. With $N_{n w}, N_{w}$ and $N_{p c k}$ fixed, change $P_{c f}$ and $P_{c b}$ (neither is directly measured) to matched the results of the six other experiments of this study.

e. If the $P_{c f}$ points scatter on the $P_{c f} \sim K$ plot, change $k_{r n w}$ at the $S_{w f}\left(k_{r n w}\right.$ has been roughly estimated before) to obtain a smoother distribution. 
If the post contact performance still has the same positive or negative errors within the comparable stages, use of a multiple-term formula instead of parabolic expressions should be considered. (However, such errors were not found in the present study).

\section{Task 4. Network/numerical model and new imbibition data.}

\section{Introduction}

New imbibition data with matched liquid viscosities and the all faces open boundary condition at very strongly water-wet conditions were presented in the preceding quarterly report. That data set was expanded to the two ends open boundary condition (radial flow) with matched liquid viscosities at very strongly water-wet condition and is documented in this quarterly report. Matched liquid viscosities had a viscosity ratio close to unity and ranged from 4 to $172 \mathrm{cp}$. The relationships between oil recovery and imbibition time are closely comparable to the results for the all faces open boundary condition. The imbibition curves were closely correlated over the entire viscosity range. The oil recovery curves exhibited essentially the same shape. Final oil recoveries showed no clear overall trend with matched viscosity

\section{Experimental}

\section{MATERIALS}

The same liquids and sandstone cores as utilized in the previously reported set with matched viscosities and the all faces open boundary condition were used for all tests in this experimental set. The preparation steps were also identical to the aforementioned experimental set.

The two ends open boundary condition (hereafter described as TEC) entails radial fluid flow. The characteristic length, $\mathrm{L}_{\mathrm{c}}$, for each core is then defined by (Zhang et al., 1996)

$$
L_{C}=\frac{d}{2 \sqrt{2}}
$$

and was close to $1.33 \mathrm{~cm}$ for each core as a result of the identical size and geometry. Oil production as a function of time was measured in standard imbibition cells at ambient temperature and with $0 \%$ initial water saturation for each test.

\section{Results and Discussion}

Imbibition results for matched viscosity were correlated by the scaling group proposed by Mattax and Kyte,

$$
t_{D, M K}=t \sqrt{\frac{k}{\phi}} \frac{\sigma}{\mu_{w}} \frac{1}{L^{2}}
$$

where $\mathrm{L}=\mathrm{L}_{\mathrm{c}}$ 


\section{SPONTANEOUS IMBIBITION}

The scaling equation of Mattax and Kyte was proposed for the special case of matched viscosities. As for the all faces open boundary condition, the equation was tested for a constant viscosity ratio very close to unity for a wide range of matched viscosities and radial flow.

Table 4.1 lists the core and liquid properties used for this experimental set.

\begin{tabular}{|c|c|c|c|c|c|c|c|c|c|c|}
\hline \multirow{2}{*}{$\begin{array}{r}\text { Table } 4.1 \\
\text { Core \# }\end{array}$} & \multicolumn{10}{|c|}{$\begin{array}{l}\text { Rock and Fluid Properties, Matched Viscosities } \\
\text { Boundary Condition: Two Ends Closed (TEC) }\end{array}$} \\
\hline & $\begin{array}{l}\mathrm{L}_{\mathrm{C}} \\
\mathrm{cm}\end{array}$ & $\begin{array}{l}\mathrm{k}_{\mathrm{g}} \\
\mathrm{md}\end{array}$ & $\begin{array}{l}\Phi \\
\%\end{array}$ & $\begin{array}{c}\sigma \\
\mathrm{dyn} / \mathrm{cm}\end{array}$ & $\begin{array}{l}\rho_{\mathrm{ap}} \\
\mathrm{g} / \mathrm{cm}^{3}\end{array}$ & $\begin{array}{l}\mu_{\mathrm{ap}} \\
\mathrm{cp}\end{array}$ & $\begin{array}{c}\rho_{\mathrm{o}} \\
\mathrm{g} / \mathrm{cm}^{3}\end{array}$ & $\begin{array}{l}\mu_{\mathrm{o}} \\
\mathrm{cp}\end{array}$ & $\Psi$ & $\begin{array}{l}\mu_{\mathrm{m}} \\
\mathrm{cp}\end{array}$ \\
\hline C1-34 & 1.321 & 77.8 & 17.4 & 39.0 & 1.119 & 4.3 & 0.782 & 4.0 & 1.09 & 4.1 \\
\hline C5-15 & 1.342 & 67.0 & 17.6 & 35.3 & 1.170 & 13.7 & 0.817 & 13.2 & 1.04 & 13.4 \\
\hline C3-3 & 1.321 & 69.9 & 17.3 & 33.9 & 1.187 & 22.5 & 0.828 & 21.8 & 1.03 & 22.2 \\
\hline C1-14 & 1.324 & 68.0 & 17.4 & 33.5 & 1.204 & 43.5 & 0.842 & 43.8 & 0.99 & 43.6 \\
\hline C5-14 & 1.329 & 75.1 & 17.6 & 32.4 & 1.212 & 59.3 & 0.851 & 59.0 & 1.01 & 59.2 \\
\hline C5-3 & 1.330 & 76.7 & 17.6 & 31.1 & 1.220 & 80.0 & 0.858 & 80.1 & 1.00 & 80.1 \\
\hline C3-31 & 1.346 & 64.3 & 16.3 & 30.5 & 1.226 & 99.7 & 0.863 & 99.1 & 1.01 & 99.4 \\
\hline C5-7 & 1.331 & 81.6 & 17.6 & 30.1 & 1.231 & 140.0 & 0.869 & 141.7 & 0.99 & 140.8 \\
\hline C3-35 & 1.347 & 71.1 & 17.8 & 30.3 & 1.235 & 171.2 & 0.873 & 173.0 & 0.99 & 172.1 \\
\hline
\end{tabular}

Fig. 4.1 shows the relationships between oil recovery and imbibition time. As the viscosity increases the rate of oil recovery systematically decreases (Fig. 4.1a). A close correlation of results over the entire viscosity range was obtained with the Mattax and Kyte scaling group (Fig. 4.1b). As for the all faces open boundary condition the shapes of the correlated oil recovery curves were all the same and no distinct trend with respect to absolute values of viscosity was recognizable. Final oil recoveries showed no clear trend with matched viscosity (Fig. 4.1c), and all data points fell within $52 \% \pm 1.5$. 

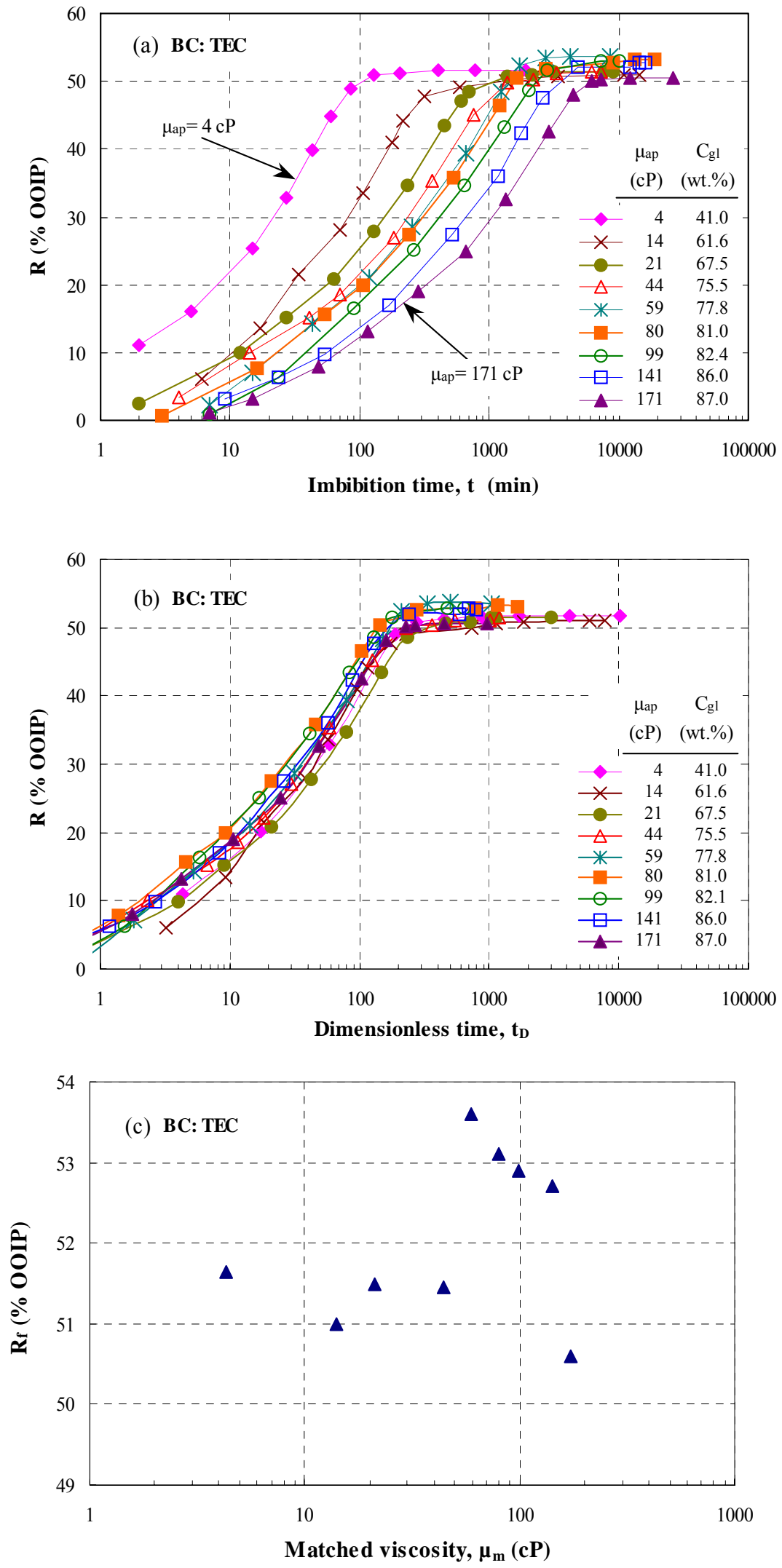

DE-FC26-03NT15408
Fig. 4.1 Recovery of oil by spontaneous imbibition for matched phase viscosity and the two ends open (TEC) boundary condition versus (a) time and (b) dimensionless time; (c) final oil recovery, $R_{f}$, versus matched viscosity. 
The dependency of the imbibition time for fractions of normalized final recovery ranging from 20 to $98 \%$ for all matched viscosity experiments are shown in Fig. 4.2a. The curves are characterized by a close to parallel alignment with increase in recovery. Fig. $4.2 \mathrm{~b}$ displays the dimensionless time for a specific recovery as a function of matched viscosity. The curves fall close to horizontal straight lines, which is another indication of close correlation.

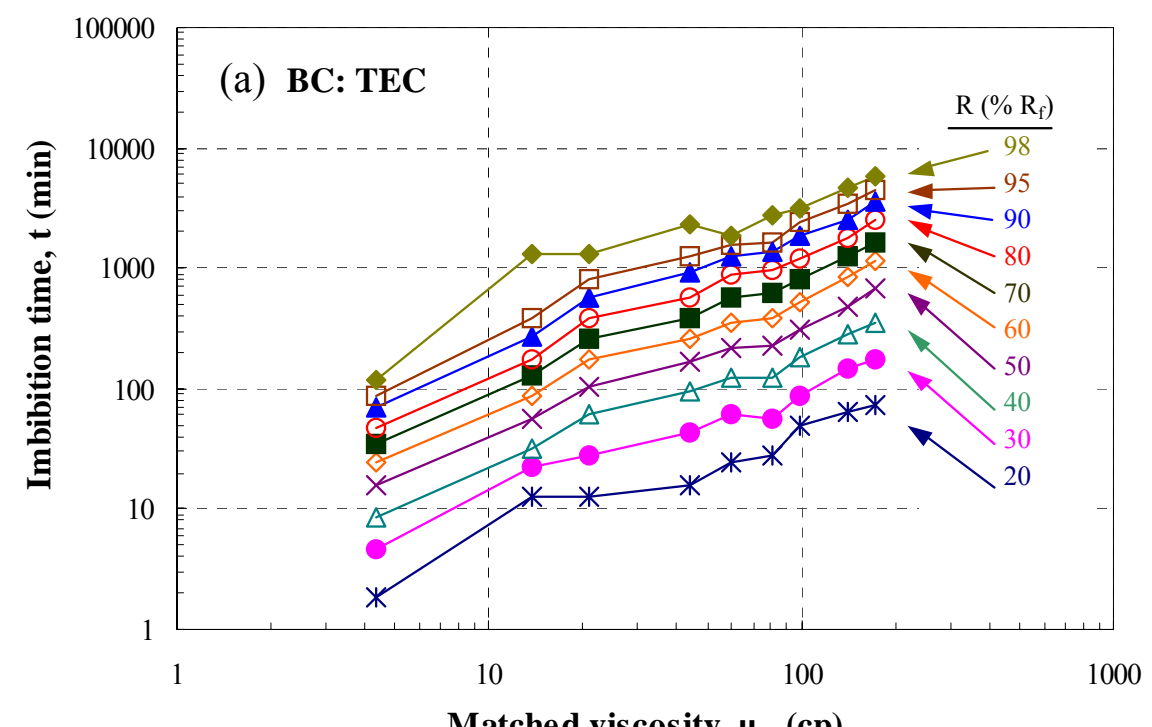

Matched viscosity, $\mu_{m}(\mathbf{c p})$

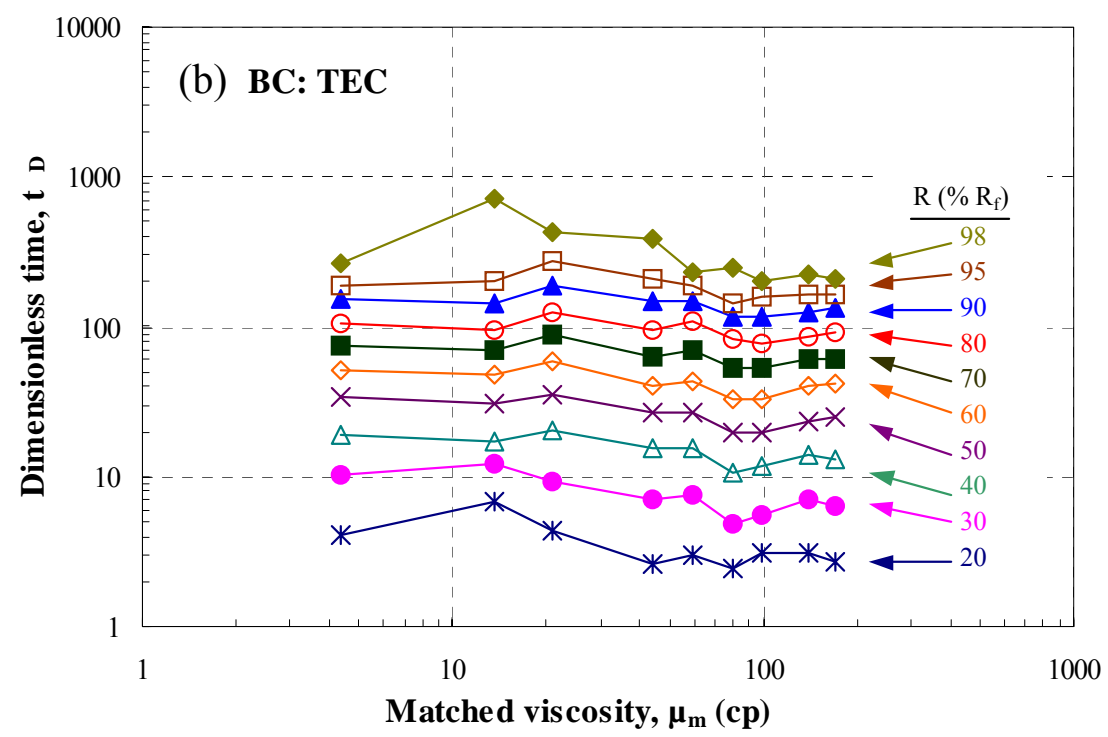

Fig. 4.2. Imbibition time (a) and scaled time (b) versus matched viscosity for fractional recoveries, R, ranging from $20 \%$ to $98 \%$ (interpolated values for individual cores). 


\section{Conclusion}

A close correlation of the imbibition data was obtained for matched viscosities (the special case of constant viscosity ratio of unity) ranging from 4 to $172 \mathrm{cp}$ and radial flow (two ends open boundary condition) by the Mattax and Kyte scaling group.

Task 5. Comparison with similarity solutions.

The objective of Task 5 is to compare results given by simulation with special case analytic results given by similarity solutions for spontaneous imbibition for at least five distinct cases of rock and fluid properties. Earlier reported findings (R01 - R05) have been rewritten and accepted for publication in Transport in Porous Media. The complete revised paper will be included in the next quarterly report.

\section{CONCLUSIONS}

1. Mercury injection data obtained for the sandstone have been used to determine work of displacement as a function of saturation. Data needs to be smoothed for change in the pressure cell and for entrance effects.

2. A dynamic network model has been coded for water/oil and liquid/gas displacements. Detailed features of the displacement mechanism including local pressure fluctuations and local flow mechanisms can be modeled.

3. Countercurrent spontaneous imbibition measurements for oil/water and air/oil, including end pressures could be closely simulated by a single set of relative permeability and capillary pressure curves.

4. Spontaneous imbibition data for matched viscosities was closely correlated by the Mattax and Kyte scaling group.

5. Experimental results and numerical simulation of one dimensional countercurrent imbibition can be closely matched by approximate analytic solution.

\section{REFERENCES}

Alpak F.O., Lake L.W. and, Embid S.M., "Validation of a modified Carman - Kozeny equation to model two phase relative permeabilities", SPE (56479) Annual Technical Conference and Exhibition, Houston, TX, $3-6^{\text {th }}$ Oct 1999.

Blair, P.M., "Calculation of Oil Displacement by Countercurrent Water Imbibition", SPEJ, Sept. 1964, 195-202.

Blunt, M.J.: "Pore Level Modeling of the Effects of Wettability," SPEJ, Dec. 1997, 2, 494-510.

Chatzis, I., Morrow, N. R.,. Correlation of capillary number relationships for sandstone, Soc. Pet. Eng. J., 1984 pp. 555-562.

Dixit, A.B., McDougall, S.R., Sorbie, K.S., and Buckley, J.S.: "Pore Scale Modelling of Wettability Effects and their Influence on Oil Recovery," SPEREE, Feb. 1999, 2, 25-36. 
Fulcher R. A. Jr., Ertekin, T., Stahl, C. D., Effect of capillary number and its constituents on twophase relative permeability curves, Journal of Petroleum Technology. February1985, pp. 249260.

Ma, S., Mason, G. and Morrow, N.R.: "Effect of Contact Angle on Drainage and Imbibition in Regular Polygonal Tubes," PS-1191, Colloids and Surfaces, 117, 1996, 273-291.

Mason, G., "A Model of the Pore Space in a Random Packing of Equal Spheres”, J. Colloid Interface Sci., 35, 1971, 279-287.

Mason, G., Nguyen, M.D., and Morrow, N.R.: "Effect of Contact Angle on the Meniscus Between Two Equal Contacting Rods and a Plate," J. Coll. Inter. Sci., 1983, 95, 494.

Mason, G. and Morrow, N.R.: "Meniscus Displacement Curvatures of Perfectly Wetting Liquid in Capillary Pore Throats Formed by Spheres," J. Coll. Inter. Sci., Jan. 1986, 109, 46-56.

Mason, G. and Morrow, N.R.: "Meniscus Configurations and Curvatures in Non-Asymmetric Pores of Open and Closed Uniform Cross-Section," Proc. Royal Soc. London, 1987, A414, 111-33.

Mason, G. and Morrow, N.R.: "Capillary Behavior of a Perfectly Wetting Liquid In Irregular Triangular Tubes," J. Coll. Inter. Sci., Jan. 1991, 141, 262-74.

Morrow, N.R., "Physics and thermodynamics of capillary action in porous media", I\&EC 1970

Morrow, N.R., and Mason, G., Recovery of oil by spontaneous imbibition, Curr. Opin. Colloid and Interface Sci., 2001, 6, pp.321-337.

Øren, P.-E., Bakke, S., and Arntzen, O.J.: "Extending Predictive Capabilities to Network Models," SPEJ, Dec. 1998, 324-336.

Pooladi-Darvish, M. and Firoozabadi, A., "Experiments and modeling of water injection in water-wet fractured porous media", J. Can. Pet. Tech., March 2000, 39, 3:31-42.

Wardlaw, N.C., Taylor, R.P., "Mercury capillary pressure curves and the interpretation of pore structure and capillary behavior in reservoir rocks", Bull. Can. PETR. Geol. 24, 1976, pp. 225262.

Washburn, E.W., “The dynamics of capillary flow”, Phys., 1921,7, 115 - 116

Zhang, X., Morrow, N.R., and MA, S., "Experimental verification of a modified scaling group for spontaneous imbibition”, SPE Reservoir Engineering, Nov. 1996, pp.280-285. 